\title{
Device and operation mechanism for non-beacon IEEE802.15.4/Zigbee nodes running on harvested energy
}

\author{
J. Pedro Amaro ${ }^{\mathrm{a}, \mathrm{b}, *}$, Rui Cortesão ${ }^{\mathrm{b}}$, Fernando J.T.E. Ferreira ${ }^{\mathrm{a}, \mathrm{b}}$, Jorge Landeck ${ }^{\mathrm{c}}$ \\ a Department of Electrical Engineering, Polytechnic Institute of Coimbra (IPC/ISEC), Portugal \\ ${ }^{\mathrm{b}}$ Institute of Systems and Robotics, University of Coimbra, Portugal \\ ${ }^{\mathrm{c}}$ ISA - Intelligent Sensing Anywhere, S.A., Rua Pedro Nunes, Edificio D, Coimbra, Portugal
}

\section{A R T I C L E I N F O}

\section{Article history:}

Received 5 February 2014

Received in revised form 10 October 2014

Accepted 24 October 2014

Available online 13 November 2014

\section{Keywords:}

Energy harvesting

Split-core current transformer

IEEE802.15.4

Zigbee

Protocol

Wireless Sensor Networks

\begin{abstract}
A B S T R A C T
The power supply is one of the main constraints when operating Wireless Sensor Networks (WSN). The most obvious energy source for a WSN node is a rechargeable or non-rechargeable battery. Batteries seriously limit WSNs usage and are associated to the increasing cost of large networks. Low cost networks will be achieved when battery consumption becomes substantially reduced or eliminated. Within this scope, powering WSN devices using mains power, is not an advantage since node installation requires complex and time consuming actions. Replacing batteries is therefore an important topic even in environments with mains power availability. In this paper, a battery-less device running an IEEE802.15.4/Zigbee protocol stack on harvested energy is proposed. Its operation mechanism and required software adaptation to sustain this complex protocol are described. The protocol compliant node uses a contact-less scavenger system that is able to power a WSN node from mains power lines thus providing an inexpensive and easily installable device even for nonskilled users.
\end{abstract}

(c) 2014 Elsevier B.V. All rights reserved.

\section{Introduction}

The energy source is the main constraint when operating Wireless Sensor Networks (WSN) [1,2]. The most obvious energy source for a WSN node is a primary or secondary battery (i.e. rechargeable or non-rechargeable). However, batteries have a number of drawbacks that seriously limit the usage of WSNs, and are in fact the main limitation to their widespread use. The WSN implementation/operation cost can be significantly reduced if node battery consumption is substantially reduced or eliminated. Eliminating batteries is an advantage in environments such as industrial platforms or large commercial and service buildings. Pres-

\footnotetext{
* Corresponding author at: Department of Electrical Engineering, Polytechnic Institute of Coimbra/Institute of Engineering (IPC/ISEC), Rua Pedro Nunes, Coimbra, Portugal.

E-mail address: amaro@isec.pt (J.P. Amaro).
}

ently, due to the high competitiveness levels worldwide in large modern industrial plants, the overall reliability and the effective maintenance management are critical aspects. For instance, when using a large number of three-phase squirrel-cage induction motors, the implementation of integrated motor monitoring and diagnosis systems is highly desirable [3]. The same applies to distributed consumption monitoring in large commercial buildings for energy management purposes. In these cases, there is plenty of available energy supply, but installing a node may require wire cutting and/or screw fastening. Moreover, maintenance tasks are frequently executed without stopping machine operation and thus performed under high power load. Connecting a WSN node to a power line in industrial environments therefore requires a skilled technician to execute hazardous operations. Home automation provides a different scenario with similar considerations. Monitoring a large number of electrical charges in 
large office buildings requires the installation of a significantly large number of wireless devices. Again in this case, despite local power availability, the use of devices with no need for maintenance may decrease operation costs. Together with energy source, WSN protocol operation is a fundamental issue. Data must be identified and related to its origin, network traffic must be routed and a network topology must be put into operation. Nodes must follow a protocol to be part of the network and are required to execute bidirectional communications. Moreover nodes must be able to listen before transmitting thus requiring more energy than if they were only sending a small number of bytes. Protocol operations such as registering with the network or joining to a group of nodes within the network are required. Protocol requires energy availability that is strongly correlated to protocol complexity.

In this paper, a battery-less device running an IEEE802.15.4/Zigbee node powered by an electromagnetic harvesting source using a Split-Core Toroidal Coil Current Transformer (SCCT) is proposed. System design options and operational analysis are addressed.

This paper is organized as follows: In Section 2 a review of energy harvesting solutions and related work is presented. Section 3 describes IEEE802.15.4/Zigbee data frame format characteristics that are relevant for the developed device, including also operational timing IEEE802.15.4/Zigbee mechanisms and related energy consumption. The implemented system architecture, hardware characteristics and capacitor based operation of the proposed device are described in Section 4. Original and proposed software adaptation of Texas Instruments Zigbee stack (Z-Stack) are described in Section 5. The proposed changes implement a task scheduler that prevents system catastrophic power down. The task scheduler algorithm is analyzed in Section 6. Conclusions are in Section 7.

\section{Energy harvesting devices, technologies and related work}

Battery replacement can only be achieved by power energy scavenger systems that, to this date, present severe limitations. Energy harvesting is the process by which energy is collected and stored from the environment. Energy can be captured from a number of sources. Solar power, salinity gradients, thermal energy, kinetic energy, wind energy, nuclear radiation and radio frequency are some possibilities to scavenge energy to power embedded systems [4-9]. Photovoltaic solutions are perhaps the most obvious power source for WSN nodes. Photovoltaic panel powering can be differentiated into two profiles as they are placed outdoor or indoor. Outdoor solar energy harvesting presents a large variation of scavenged power as day light varies from a clouded to a sunny day. Indoor variation are due to different light sources and different illumination profiles [4,10-16]. Mechanical force can generate energy if an inertial mass is used to create movement. Movement can be converted into electric energy using three mechanisms: electrostatic, piezoelectric and electromagnetic [4,17-19]. A vibrating piezoelectric device is used to convert mechanical energy from pressure, vibra- tions or force into electricity. The piezoelectric harvester consists of a capacitor formed by one or several piezoelectric layers sandwiched between metallic electrodes. The principle of operation of the energy scavenger is based on the mechanical strain exerted on a piezoelectric mass. Electrostatic energy harvesting is done by changing the capacitance of a variable capacitor. This mechanism uses plate vibration of a previously charged capacitor to produce electrical energy. Promoting changes on a magnetic field through mechanical vibration can be characterized as electromagnetic energy harvesting. An electromagnetic induction is therefore achieved by using a permanent magnet, a coil and a mechanical device. Thermal energy harvesters are based on the Seebeck effect, which states that a voltage is created at the junction of two different metals or semiconductors if a temperature difference exists between both plates. The core element of a thermal energy harvester is the thermopile, which is a device formed by a large number of thermocouples placed between a hot and a cold plate and connected thermally in parallel and electrically in series [20-23]. Radio frequency is identified as a possible energy harvesting source for embedded systems. RFID $^{1}$ is a common technology in which a small circuit is powered by radio frequency bursts. The exact same principle can be adopted by WSN nodes as a radio frequency burst can be used to power a group of nodes [10,24-27]. A nuclear diode junction battery is presented in [28-31]. This work suggests a $50+$ year lifelong energy source with $1.5 \mathrm{~V}$ voltage value and currents of nano to microampere. The used $63 \mathrm{Ni}$ isotope is a device that directly converts nuclear to electric energy with a very low alpha radiation emission. In general, the output of an energy harvester cannot be used to directly power embedded system circuits $[10,32,16]$. Conversion circuits must therefore be used to accommodate voltage and power levels. Power management circuits must be able do adapt to harvester conditions and characteristics. The variability in voltage, power density, and duty cycle requires careful design of conversion circuitry to effectively collect harvested energy. The power management unit should be able to handle very low feeding power and should also be self-starting.

The work presented in [33] reviews and compares available options for powering WSN nodes from large electric and magnetic fields which exist near high-voltage electrical installations such as substations. In [34] a microprocessor controlled electromagnetic energy harvesting device from a single high voltage transmission line is presented. This system is able to scavenge energy from line currents between $65 \mathrm{~A}$ and $130 \mathrm{~A}$. A magnetic power generator and a voltage multiplier are used. In [35] a communication concept for inverter fed electric motors is presented. Power-line communication is used to send motor operational data. Power supply is obtained by inductive coupling from the remaining motor phases. In [36] a small electromagnetic energy harvesting device is proposed, where several topologies for the magnetic coupler are tested. This energy harvesting device is used to charge a battery that can in turn power a wireless device. These works investi-

\footnotetext{
1 RFID: Radio-Frequency Identification.
} 


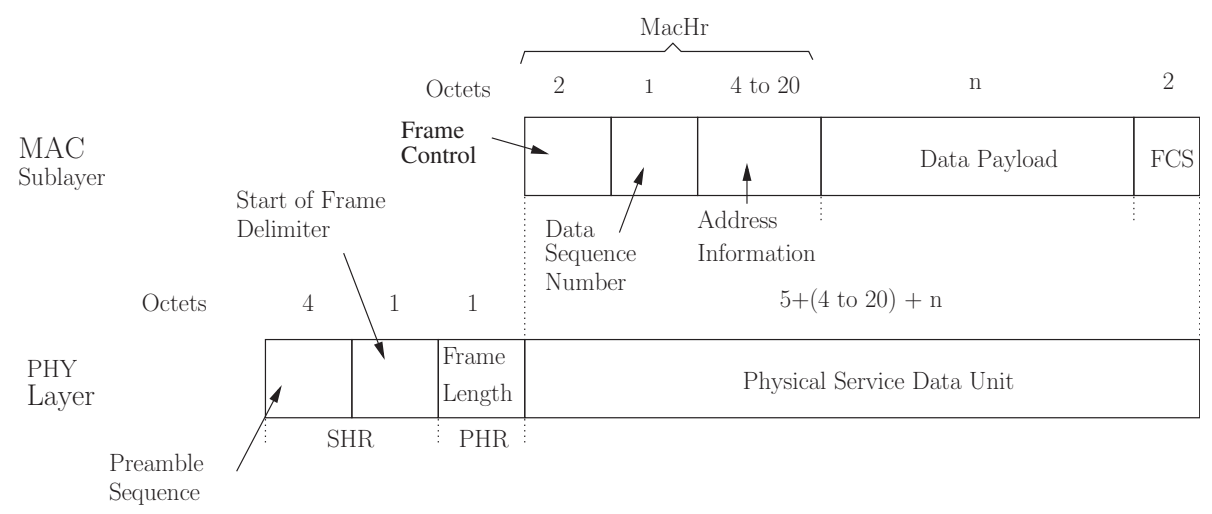

Fig. 1. IEEE802.15.4 data frame structure.

gate the harvesting ability of the proposed devices and no mention is found that relates protocol complexity with the developed devices. The protocol implication of running a WSN node on harvested energy is investigated in $[37,38]$. The presented results determine the optimal amount of energy to be drawn from the storage devices given the knowledge of the state of charge (SOC). Moreover, the impact of defective SOC estimation is computed and the harvested energy addressed as a random variable. This work assumes the microcontroller to be the heart of the energy management process but addresses its operation using a generic approach. Also in [39,40], solutions are presented that may be identified as related to the work presented in this paper. In [39] a harvesting device running on capacitor stored energy is presented. This device presents a software controlled mechanism that maximizes the nodes sensing and communication. The system scavenges energy from the environment radio frequency and accounts for capacitor leakage currents to control the sensing and communication mechanisms. In [40] a device is presented that is able to scavenge energy from high and medium voltage power lines electric fields. Simulation and an implemented DC regulator are presented. Our early work introduces the protocol complexity as an important limit to the harvesting device operation. Moreover, microcontroller and operating system behavior may have fundamental importance for a device powered on scavenged energy. The Split-Core Current Transformer has been successfully applied in [41] to power a battery-free wireless device running on harvested power that is capable of monitoring three-phase squirrel-cage induction motor parameters. In [41], the device is unable to power the protocol initialization procedure and is therefore deployed after initialization. The system presents little survivability time when mains current fails. The device proposed in this paper stands for an important evolution as it is fully protocol compliant and largely extends device lifetime.

\section{IEEE802.15.4/Zigbee operation}

\subsection{IEEE802.15.4/Zigbee data frame structure}

IEEE802.15.4/Zigbee is a well specified, studied and described standard in literature. Moreover, a number of protocol stack implementations are freely distributed [42-44], making it a very useful tool for test and verification of WSN with an added characteristic of small time to market implementation. IEEE802.15.4/Zigbee is nevertheless a protocol specification with a large number of options thus making software implementations large and complex. To the purpose of this work the choice of this complex protocol is justified by the fact that if the system is able to operate with it, it will also be able to operate with a different and simpler protocol. A carrier sense for multiple access with collision avoidance (CSMA/CA) $[45,46]$ mechanism is used by the radio for medium access. Medium Access Control (MAC) data transactions are executed using four frame structures: Beacon frame, transmitted by a Zigbee Coordinator (ZC) for network information and synchronization; data frame, used for data transfers; Acknowledgment frame, used at various levels to confirm successful reception; MAC command frame, used for handling all protocol entity control transfers (IEEE802.15.4 MAC commands). Fig. 1 shows the IEEE802.15.4 data frame structure with particular relevance to its size in number of bytes. In this figure SHR and PHR are the Synchronization Header and the Frame Length for the Physical Layer and $\mathrm{MaCHr}$ is the MAC Layer Header. Even though non-periodic operation can be used, the IEEE802.15.4 protocol is well suited for periodic operation mode for all network participants.

\subsection{IEEE802.15.4/Zigbee registration and communication procedures}

Wireless networks nodes are microcontroller based devices. If low power based operation is required, microcontroller devices must be able to enter a mode of low current consumption. Zigbee End Devices (ZED) make use of the same mechanisms to lower drawn current. When entering in sleep mode, the processor shuts down the high frequency oscillator together with peripherals and processor core. In a disconnected operation the device only joins to the network when it needs to communicate. Connected operation mode requires that the Join procedure $[47,48]$ is executed once by all nodes. By joining the network, a device is associated to another device that is already part of the network. A device that has multiple devices associated with 




Fig. 2. 50-s measurement of a ZED device operating a Zigbee compliant stack illustrating absorbed current. Obtained with an experimental setup, measuring current consumption of a ZED in a simple two node network. The setup measures the voltage drop on a $1 \Omega$ resistor in series with the CC2530 microcontroller voltage line.

it is a coordinator or parent of those devices. Prior to joining the network each node must execute a network scan, thus choosing a suitable parent using design parameters. After potential parent identification, nodes issue an association request command frame. Parents must then determine if requesting nodes can join, sending a response frame in accordance. If join is successful, the response frame contains the short address that the device has to be identified within the network. This procedure entails an association request action from the ZED as well as an Acknowledgement (Ack) reception. After a aResponseWaitTime [47] delay period a data request is executed to determine if the join request is accepted. The answer is issued using an association response. ZEDs may sleep after sending the join request. The aResponseWaitTime is a IEEE802.15.4 defined MAC specification attribute that determines the maximum time a device shall wait for a response to a command frame. The fixed-time aResponseWaitTime is determined by $32 \times$ aBaseSuperframeDuration [47,49], thus entailing a

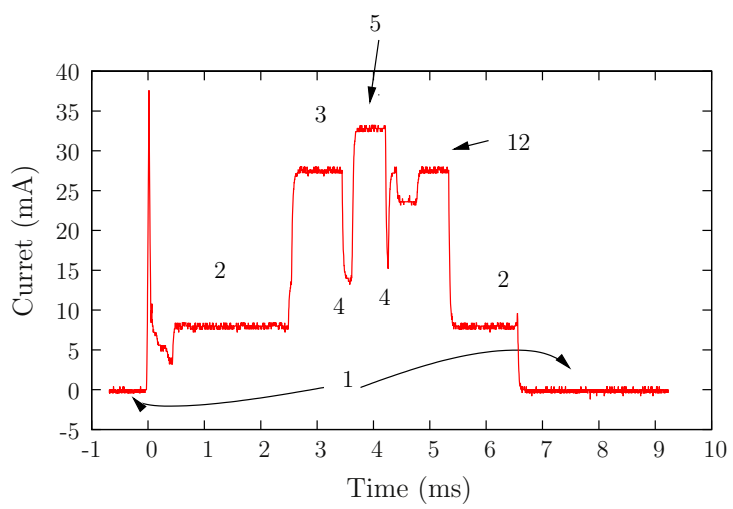

(a) Drawn current during data requests with MAC layer acknowledgement.
491.52 ms wait time. Active Scans $[47,50]$ consist on a sequence of beacon requests, using MAC frame commands, to determine the existence of network coordinators or routers using different radio channels. This operation is executed with radio frequency module turned on for each channel scan a period of time ScanDuration. Network joining may require a prolonged turn-on time for the radio frequency module being therefore energy consuming. Join procedure is however fundamental for protocol operation as nodes use it to acquire a valid network address. Nodes with linked functionalities share common application object structures. The process that relates two node application objects is described as binding.

A ZED node full operation with Active Scan/Join, Bind and data transfer actions is illustrated in Fig. 2. As illustrated, a data request leads to less energy consumption than the Join/Active Scan procedures. Moreover, within the shown 50-s test, energy consumption of joining the network using an Active Scan procedure is the system main energy constraint. All procedures are seen as contributors to battery exhaustion. In fact, usual power management analysis, frequently address Active Scan, Join and Bind operations as over time small contributors to energy exhaustion. This is due to the fact that a large number of small but regular low power data transfers have a larger impact on battery lifetime than few high current consumption actions. Nodes powered on harvested energy that rely only on capacitors for system operation must address these protocol operations differently. In this work, adaptation on the hardware side is done by dimensioning energy storage capacitors to comply with Join and Active Scan actions. On the software side, all procedures are executed only if enough energy is available, returning the processor into sleep mode immediately after.

\subsection{Understanding current consumption of IEEE802.15.4/ Zigbee operation}

In this work, a device using a Texas Instruments CC2530 [51] microcontroller with a radio frequency module for



(b) Drawn current during data requests with Application layer acknowledgement.

Fig. 3. Drawn current for regular data transfers on ZED. Obtained with our setup, measuring current consumption of a ZED in a simple two node network. The setup measures the voltage drop on a $1 \Omega$ resistor in series with the CC2530 microcontroller voltage line. 
2.4-GHz IEEE802.15.4 and ZigBee applications implements a processing and communication system. The system is programmed using Texas Instruments Zigbee protocol (ZStack [42]).

Unlike battery powered nodes, a device running exclusively on capacitor charge can easily run into power failure. This event occurs if the microprocessor power line, in this case the CC2530, lowers under operational limits. In [51] this voltage limit is announced to be $1.8 \mathrm{~V}$. Node operation is highly parameterizable and a number of options are available in Z-Stack. Zigbee operation and concomitant power consumption is not however exclusively dependent on programming. Fig. 3 shows drawn current for two operational modes for Zigbee data transfers.

Fig. 3a illustrates drawn current during data requests with MAC layer Acknowledgement. This figure shows the drawn current for a frequent action executed in low-power ZED where the node polls its coordinator for application level data. Fig. 3b illustrates the same behavior with an actual data transfer and application level acknowledgement. In this case, after processing received data, the ZED sends an application level acknowledgment and waits for a MAC level acknowledgment. A data request entails a sequence of actions that are identified in these pictures by the numbering. Time slot 1 corresponds to a current related to the CC2530 power mode in which only one timer and a low frequency oscillator are running. The microcontroller wakeup time interval 2 is related with startup sequence when the microcontroller enables its $32-\mathrm{MHz}$ oscillator. The CSMA/CA algorithm is executed in time slot 3. In this slot the node executes a Clear Channel Assessment (CCA) with a backoff mechanism which presents variable time length. The CSMA/CA time slot $\left(t_{C S M A}\right)$ behavior may be described by $[52,53]$ :

$t_{C S M A}=\sum_{i=0}^{a \operatorname{MaxBO}}\left(\left(\operatorname{rand}\left(\left[0,2^{\min (a \operatorname{Min} B E+i, a M a x B E)}\right] \times t_{a U n i t B O}\right)-1\right)+t_{c c a}\right)$

where rand is a random function, $a M i n B E$ and $a M a x B E$ are the initial and maximum backoff exponents for successive carrier sensing action, $t_{\text {aUnitBo }}$ is the unit backoff period for 2.4-GHz frequency band (aUnitBackoffPeriod in IEEE802.15.4 definition), $a M a x B O$ is the maximum number of times the CSMA mechanism is repeated after the first occupied channel occurrence and $t_{c c a}$ is the time a radio module listen time for a channel assessment. Prior to data transmission, nodes execute a radio wave carrier sense to determine if other nodes are transmitting. The first Clear Channel Assessment (CCA) is executed after a random backoff period. If the radio channel is busy a new backoff period is measured until a new CCA is executed. This action is repeated $a \mathrm{MaxBO}$ times and a communication failure is reported to higher layers if the radio channel is never considered available. Time slots identified with 4 have fixed length and are related to radio module switching from receiving to transmitting mode and vice versa. Data request is transmitted in time slot 5 whose length is related to the fixed number of bytes this specific command sends. Time slot 12 is also fixed and relates to sending or receiving MAC level acknowledgment. The time to trans-

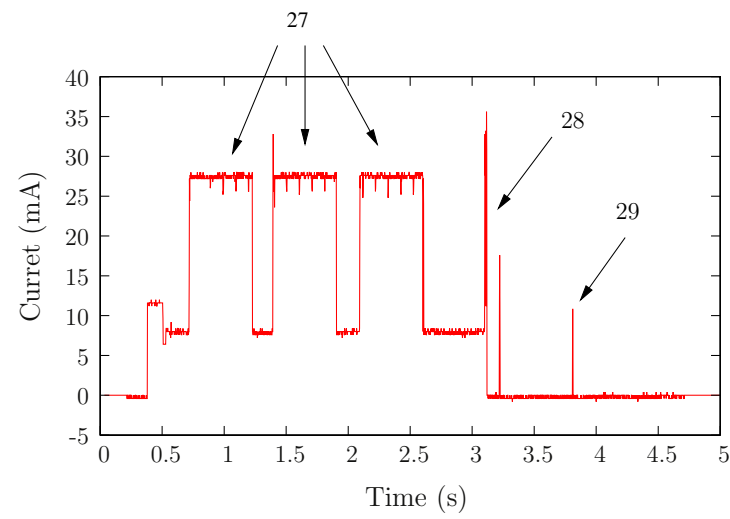

Fig. 4. Active Scan and association Zigbee device absorbed current. Obtained with our setup, measuring current consumption of a ZED in a simple two node network. The setup measures the voltage drop on a $1 \Omega$ resistor in series with the CC2530 microcontroller voltage line.

mit or receive a number of bytes $\left(t_{T x R x}(n)\right)$, illustrated by time slot 7, is given by [52]:

$t_{T x R x}(n)=\frac{8(S H R+P H R+M a c H r+n)}{d r}$

where SHR, PHR are the Synchronization Header and the Frame Length for the Physical Layer, MacHr is the MAC Layer Header, $d r$ is the data rate of $250 \mathrm{kbps}$ for the 2.4$\mathrm{GHz}$ frequency band and $n$ is the number of bytes to send or receive. SHR, PHR, MacHr are illustrated in Fig. 1. Time slot 8 shows application layer acknowledgment transmission.

In Fig. 4, Active Scan and network join procedures drawn current are shown. In non-beacon Zigbee networks a node wishing to join the network searches first for the best parent. The best parent is selected by issuing a beacon request using a command frame and listening for answers thus maintaining radio module in $\mathrm{Rx}$ mode for a period of time. This action can be repeated for several radio channels thus entailing a variable radio wake time. A node must therefore remain in active $\mathrm{Rx}$ mode for a long period of time as shown by time interval 27 in Fig. 4. This time interval $\left(t_{\text {scan }}\right)$ is dependent on IEEE802.15.4 standard defined constants:

$t_{\text {Scan }}=\sum_{\text {FistChn }}^{\text {LastChn }}$ aBaseSuperFrameDuration $\left(2^{\text {ScanDuration }}-1\right)$

where aBaseSuperFrameDuration is one of these constants and represents a base time of $15.36 \mathrm{~ms}$ in $2.4-\mathrm{GHz}$ frequency band $[47,49]$. ScanDuration is the protocol defined parameter that allows software control over scan time duration, FistChn and LastChn are respectively the first and last channel of the scanning mechanism. The time slot 28 shows the required time length to execute a join operation as described in Section 3.1. Table 1 summarizes drawn current requirements for Zigbee data exchanges for each identified time slot. 
Table 1

Data exchange drawn current profiles. Obtained with our setup, measuring current consumption of a ZED in a simple two node network. The setup measures the voltage drop on a $1 \Omega$ resistor in series with the CC2530 microcontroller voltage line.

\begin{tabular}{|c|c|c|}
\hline Slot & Time length & Current \\
\hline 1 & Variable. Time between two consecutive transmission & $1 \mu \mathrm{A}$ \\
\hline 2 & Application dependent data processing. Processor runs at $32 \mathrm{MHz}$ oscillator that requires $300 \mu$ s of start-up time & $0.76 \mathrm{~mA}$ \\
\hline 3 & CSMA/CA algorithm & $27 \mathrm{~mA}$ \\
\hline 4 & Switching $\mathrm{Tx} / \mathrm{Rx}$ or $\mathrm{Rx} / \mathrm{Tx}$ radio module & $14 \mathrm{~mA}$ \\
\hline 5 & Data request transmission. Fixed number of bytes & $33 \mathrm{~mA}$ \\
\hline 7 & Receive data from coordinator & $27 \mathrm{~mA}$ \\
\hline 12 & MAC Layer Ack & $33 \mathrm{~mA}$ \\
\hline 13 & Tx of Application layer Ack. Application dependent & $33 \mathrm{~mA}$ \\
\hline 27 & Channel scan & $33 \mathrm{~mA}$ \\
\hline 28 & Join procedure (see Section 3.2) & - \\
\hline 29 & Data request (see Section 3.2) & - \\
\hline
\end{tabular}

\section{Our solution for a harvested power IEEE802.15.4/ Zigbee device}

Powering electronic devices is usually associated with a reasonably stable power supply. Portable or other independent devices are commonly powered by batteries that provide a reasonably stable voltage level. Our battery-less device relies exclusively on capacitors to power a radio frequency module and a microcontroller operation. Unlike a battery operated device, once current is drawn from capacitor, voltage may drop thus failing microcontroller powering. In one such system, capacitor discharge control is mandatory. In this work, a battery-less energy harvesting system for a IEEE802.1.4/Zigbee module is built. The energy is harvested from a contact-less magnetic field generated by an alternate current flowing through a power line. Capacitor charge is managed so that protocol operation is possible. Fig. 5 shows the proposed device as well as the implemented system architecture that is based on the operation of three capacitors. A Split-Core Current Transformer (SCCT) is used to generate a current from the induced electromotive force. The SCCT coil is used to scavenge energy from power cables connecting loads (e.g. electric motors, lighting, computers). The LTC3108 [54] integrated circuit from Linear Technology is used to accommodate voltage and power levels as well as charge three capacitors. The LTC3108 implements a charge pump from the AC SCCT rectified voltage. The rectified voltage is used to operate a booster circuit that regulates to the desired stable output value. LTC3108 operation voltage has a stabilizing capacitor connected to $V_{a u x}$ that when it reaches $2 \mathrm{~V}$ allows output capacitor connected at $V_{\text {main }}$ to begin charging. $V_{\text {main }}$ in turn charges to the maximum voltage which for this system is set to $2.5 \mathrm{~V}$ or $3.3 \mathrm{~V}$. Once $V_{\text {main }}$ reaches the maximum voltage, $V_{\text {storage }}$ is allowed to start charging, that in turn charges up to $5.25 \mathrm{~V}$. After $V_{\text {storage }}$ full charging, excess current is shunted to ground if no load exists at device output or if the input source is generating more power than it is required by the load. $V_{\text {storage }}$ can be used as an energy reservoir to power $V_{\text {main }}$ when energy is missing. Charging is possible for mains currents as low as $500 \mathrm{~mA}$. The proposed system operation should meet energy constraints to prevent the $V_{\text {main }}$ capacitor from discharging bellow the microcontroller minimum operating voltage of $1.8 \mathrm{~V}$.

\subsection{SPICE model assessment, simulation and experimental results}

For the development of this harvesting system, a SPICE [55] model of the SCCT is implemented and the results are assessed with device measurements. The proposed SCCT SPICE model has been used together with the Linear Technology LTC3108 SPICE model to simulate system operation.

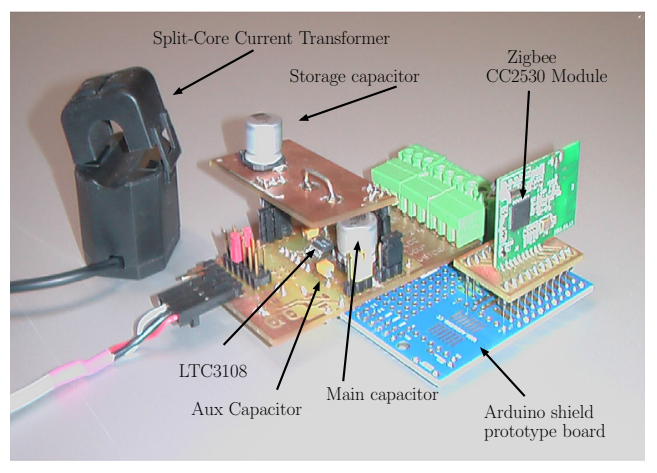

(a)

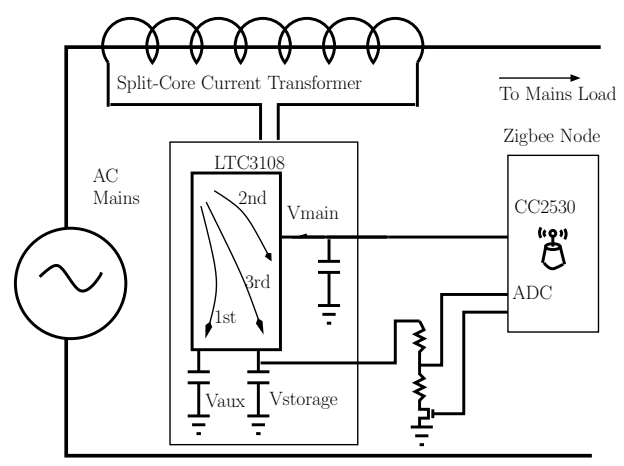

(b)

Fig. 5. Illustration of our harvested power IEEE802.15.4/Zigbee device and corresponding scheme architecture. (a) Device photo. (b) System architecture. 




Fig. 6. Simulated and measured device operation for $330 \mu \mathrm{F}$ capacitors, with 1-A and 700-mA mains current.

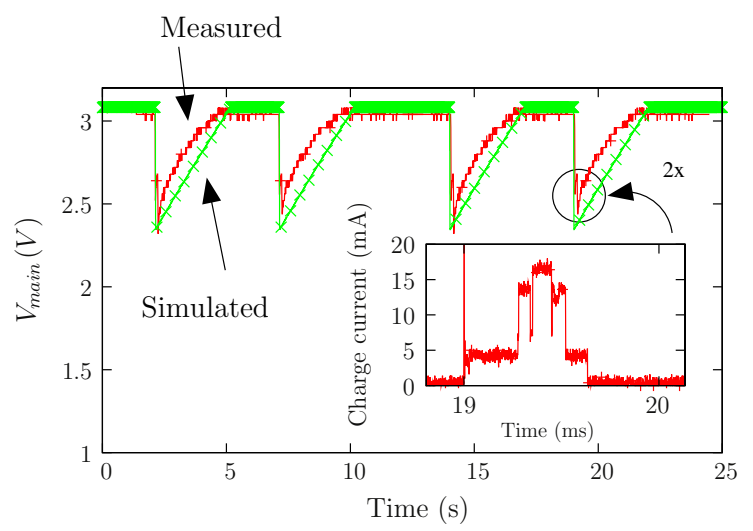

Fig. 7. System operating the Zigbee compliant stack $v_{\text {main }}$ voltage line level for $330 \mu \mathrm{F} V_{\text {main }}$ and $V_{\text {storage }}$ capacitors.

A study of the harvesting device is presented in [56]. In this work, a capacitor powered device is proposed and the simulated results illustrate that the system is able to sustain regular IEEE802.15.4/Zigbee operations (e.g. data transfers). In [57], a method is proposed to determine the characteristics of a generic Split Core Current Transformer. These characteristics are included in a SPICE simulator model. The model is compared to measured results and shown to be accurate by implementing a device that validates system operation for IEEE802.15.4/Zigbee data transfer operations. This study does not include the nodes behavior for IEEE802.15.4/Zigbee network join and scan operations. An analytic study of the proposed device is also missing from these papers. Fig. 6 illustrates both simulated and measured device operation for $1 \mathrm{~A}$ and $700 \mathrm{~mA}$ mains power line current and $330 \mu \mathrm{F} V_{\text {main }}$ and $V_{\text {storage }}$ capacitors, thus allowing SPICE model assessment. In these tests, our system has been first fully discharged and placed in operation thereafter. Also the microcontroller is programmed to enter in low-power mode immediately after power up. As shown in Fig. 6, capacitor charge waveform simulations are in accordance with measured results.

\subsubsection{Results for 10-byte data transfers}

When addressing the scenario of temporary motor monitoring, nodes may be deployed after performing Active Scan and Join procedures. During device deployment, system operation is dependent on capacitor previously stored energy because the SCCT power source is unavailable. The processor must be running in low power mode so that capacitor stored energy is not depleted and operation is therefore sustainable during the deployment time frame. This is a desirable operation mode since installing our device does not require that machines are stopped. After deployment, monitoring nodes are required to transfer their acquired data to a central node. It has been shown in [41], that a data transmission of 10 bytes is sufficient to transfer motor operational data. For this situation nodes first send a data request and transfer data thereafter thus using two data frame communications. Fig. 7 illustrates system $V_{\text {main }}$ voltage line running Z-Stack while executing 5-s spaced 10-byte data transfers. Both simulated and measured plots are shown for a system with $330 \mu \mathrm{F}$ $V_{\text {main }}$ and $V_{\text {storage }}$ capacitors. For the simulated results a piecewise average drawn current is used to model the data request shown in Fig. 7 detail. The minimum voltage $V_{\text {main }}$ is maintained over $2 \mathrm{~V}$ thus preventing microcontroller shutdown. This test shows that it is possible to maintain system regular operation for a ZED based on capacitors.

4.1.2. Results for Active Scan, Bind and 10-byte data transfers

Monitoring large buildings is a different scenario for this system application. In this context, RF communication is constrained by a number of factors like PCB printed

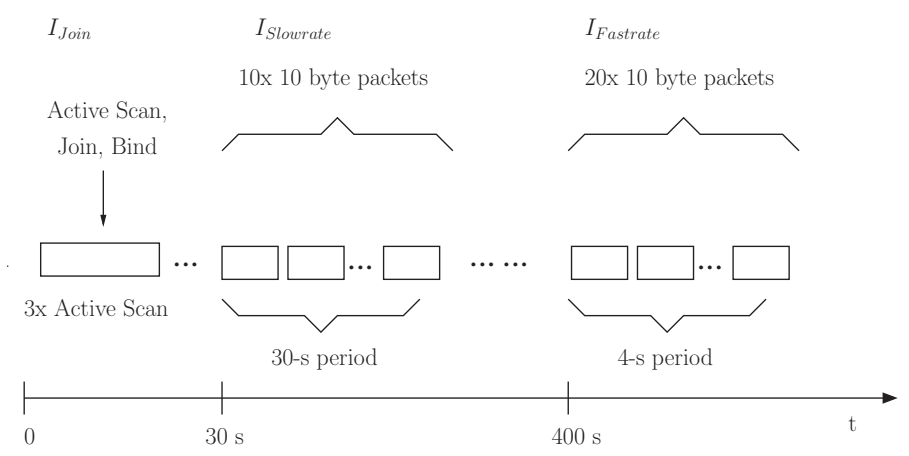

Fig. 8. Data transfer executed test description. 


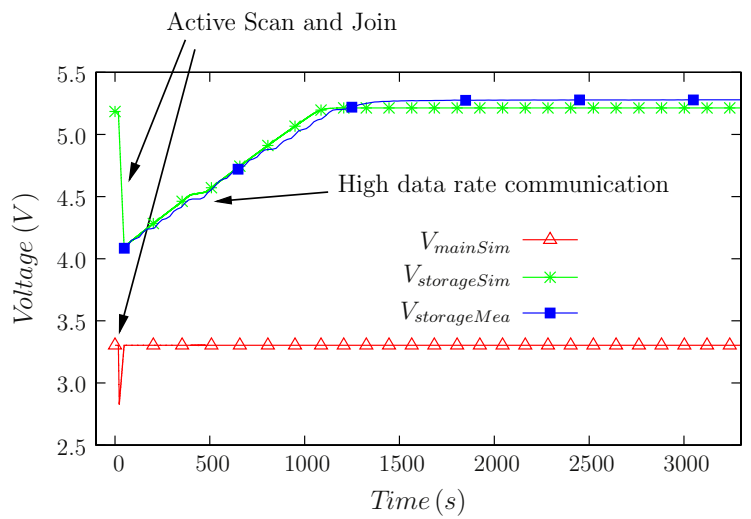

(a) One hour measured and simulated data.

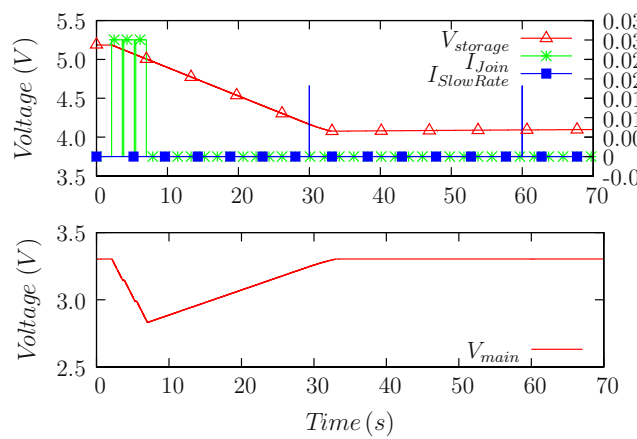

(b) Initial 70-seconds detail simulation.

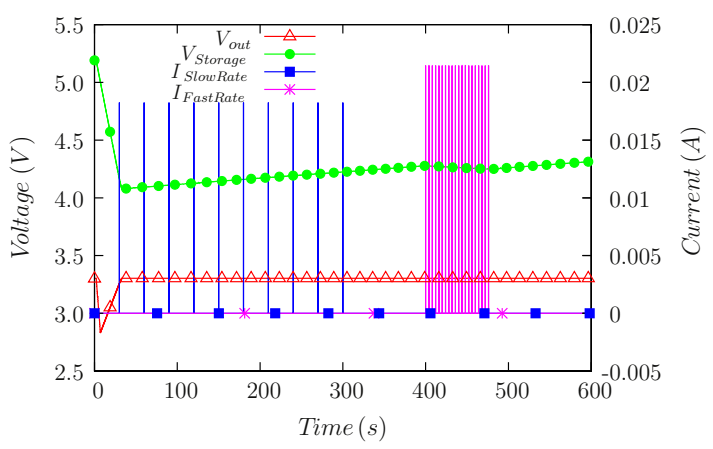

(c) Initial 600-seconds simulation.

Fig. 9. $V_{\text {storage }}, V_{\text {main }}, I_{\text {Join }}, I_{\text {SlowRate }}$ and $I_{\text {FastRate }}$ levels for Active Scan, association, Bind and data communication (a) full operation, (b) 70-s detail, (c) 600-s detail.

antennas, possibly poor RF design and thick concrete walls. Each ZED node frequently loses radio link with its parent. Also, in this case, network reconfiguration or redeployment requires that the Join procedure is executed. The device configuration described in Section 4.1.1 does not handle these events as the charge stored in $330 \mu \mathrm{F}$ capacitors $V_{\text {main }}$ and $V_{\text {storage }}$ is insufficient for a long period of radio module turn on time. Sustaining exception procedures is possible by increasing $V_{\text {main }}$ and $V_{\text {storage }}$ capacitance through supercapacitors. A $240-\mathrm{mF} V_{\text {main }}$ capacitor has

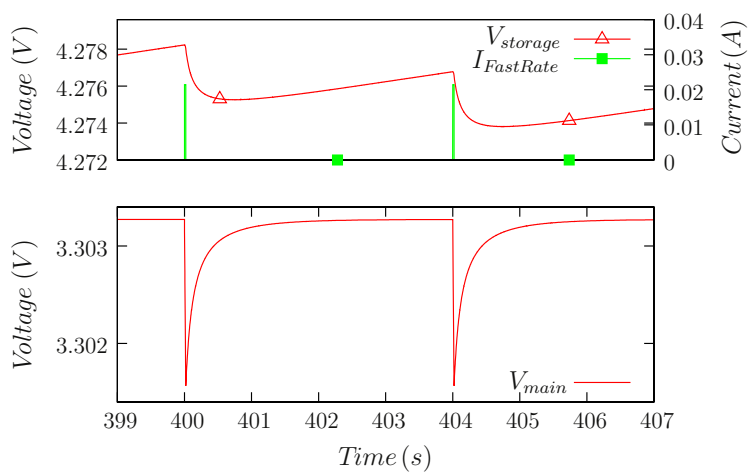

Fig. 10. Active Scan and association Zigbee device detail drawn current and voltage variation.

been chosen because of its ability to sustain Active Scan Out-of-the-Box Zstack operation. Moreover, the $V_{\text {storage }}$ $120 \mathrm{mF}$ capacitor value corresponds to two serially connected $240 \mathrm{mF}$ capacitors as the $5.2 \mathrm{~V}$ maximum voltage exceeds these components voltage rating. The system ability to sustain the full Z-Stack operation has therefore been tested. Fig. 8 describes the proposed test sequence. For this test, after execution of the Join procedure, the ZED is programmed to send 10 bytes of data with MAC level acknowledgment. These frames are transmitted with a time period of $30 \mathrm{~s}$ ending $300 \mathrm{~s}$ after the first sent frame. The current associated with these frames is named $I_{\text {slowRate }}$. ZED's parent is programmed to return data starting at $400 \mathrm{~s}$. Data are returned with 4-s period, application level acknowledgment and each data frame is sent after a ZED data request command. The current associated with these frames is referenced as $I_{\text {FastRate. }}$. Fig. 9a illustrates simulated and measured capacitor $V_{\text {main }}$ and $V_{\text {storage }}$ voltages as the system sustains Active Scan, Join and data transfers operations for a 3600-s period. The Join procedure impact on both $V_{\text {main }}$ and $V_{\text {storage }}$ is shown. $V_{\text {main }}$ discharges from $3.3 \mathrm{~V}$ down to a voltage level of $2.8 \mathrm{~V}$ that still is within CC2530 accepted values. $V_{\text {storage }}$ charge restores the $V_{\text {main }}$ capacitor and recharges thereafter. Capacitor recharge is illustrated for a 1.2 A mains current.

Figs. 9b, c and 10 illustrate zooming details of Fig. 9a. Fig. 9b shows a 70-s detailed system operation after ZED joining the network to illustrate the impact on both $V_{\text {main }}$ and $V_{\text {storage }}$ capacitors. In this figure, the drawn current associated with Active Scan, Join and Binding procedures is referenced as $I_{\text {Join }}$. Fig. $9 \mathrm{~b}$ also shows the first two $I_{\text {SlowRate }}$ occurrences, as well as the relative low influence on capacitor voltage levels. Fig. 9c details the impact of $I_{\text {SlowRate }}$ and $I_{\text {FastRate }}$ series of data communications on $V_{\text {main }}$ and $V_{\text {storage }}$ voltage levels. This detail shows that the system is able to maintain a stable $V_{\text {main }}$ voltage level for both communication data. For the $I_{\text {FastRate }}$ data series, $V_{\text {storage }}$ capacitor is required to charge $V_{\text {main }}$ thus losing its own charge, demonstrating that $I_{\text {DataFast }}$ is not long term sustainable by the device. Fig. 10 illustrates the impact of two successive 4-s period $I_{\text {FastRate }}$ drawn current on $V_{\text {storage }}$ and $V_{\text {main }}$. The impact on $V_{\text {main }}$ has an amplitude of $2 \mathrm{mV}$ that is not visible in Fig. 9c. However, the $V_{\text {storage }}$ capacitor is 


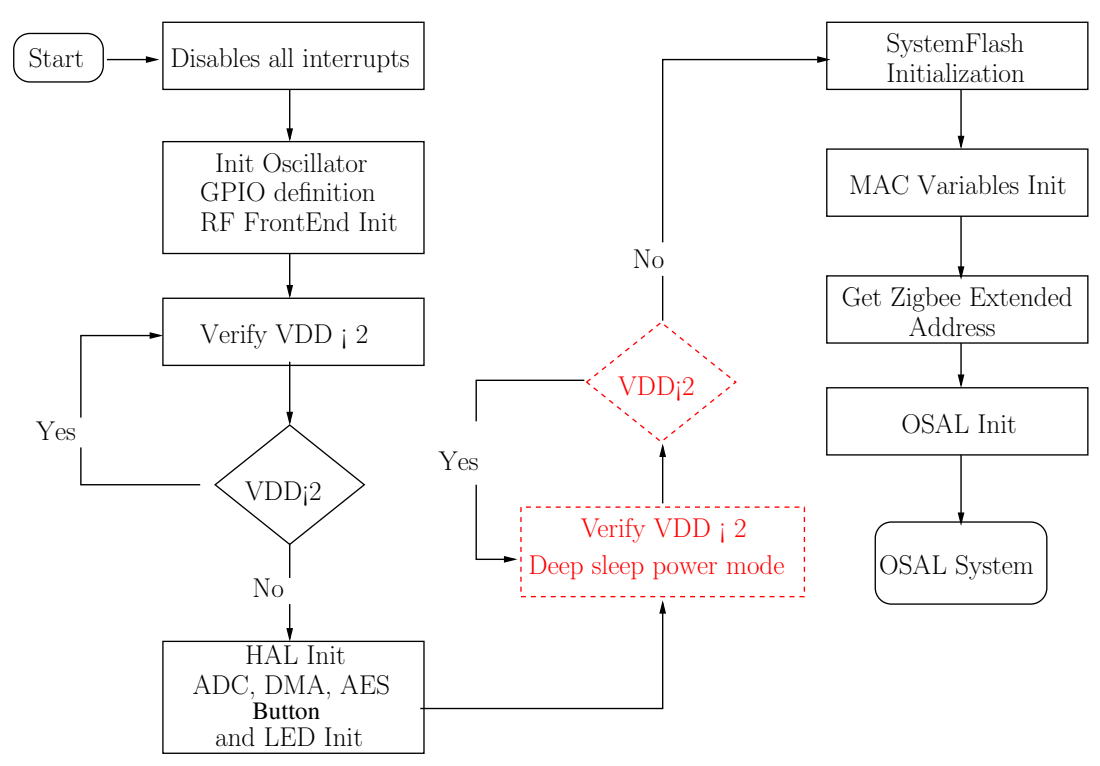

Fig. 11. Z-Stack operating system abstraction layer initialization procedure.

not able to recover and the detail of its descending voltage level is observed. The system is able to operate without microcontroller shutdown and data exchanges were executed in a noiseless environment with low probability of collision or occupied channels. The system main constraint is shown to be the Active Scan procedure, which requires the use of milli-Farad capacitors, having impact on charge times.

\subsection{Capacitor design issues}

The capacitance value is a relevant design option with critical influence on charge times. Moreover in the absence of mains current, capacitor leakage current is relevant for system lifetime even without radio frequency activity. The choice of capacitor devices is therefore limited to those with low leakage characteristics. Also devices presenting high capacitance values are usually associated with electrolytic or tantalum capacitors that present high leakage currents. Leakage capacitor current is outside the scope of this work and is addressed in $[39,58]$. As a design option for $330 \mu \mathrm{F}$ devices, Vishay Aluminum capacitors [59] are recommended by the LTC3108 datasheet. Moreover, larger capacitance values obtained from Cellergy [60] present desired characteristics of high capacity and low self discharge current.

\section{IEEE802.15.4/Zigbee task scheduler for nodes running on harvested power}

Texas Instruments Z-Stack implements an operative system that is referred to as board support package (BSP) [61]. This BSP consists of a hardware abstraction layer (HAL) and an operating system abstraction layer (OSAL). OSAL is a mechanism for task allocation of resources, implementing a cooperative, round-robin task servicing loop where each operation in Z-Stack runs as a task that is capable of communicating with other tasks through a message queue [62]. Original OSAL implementation is developed for battery operated nodes with no short term energy limitations. In [63] the main energy consumer processes within Z-Stack are identified. In [64] the changes introduced to Z-Stack so that system is able to operate the IEEE802.15.4/Zigbee data transfers are presented. Both studies relate only to data transfer operations. Moreover, none of these papers presents analytic results of the proposed mechanism. The Out-of-the-Box implementation executes Z-Stack initialization, IEEE802.15.4 Join and association procedures as fast as possible without entering low power mode and only then returning to processor sleep mode. This is a natural and desirable behavior for battery operated nodes. For such power supplies, short term energy exhaustion is not a problem and is therefore indifferent to remain on full power mode for one time 1 or $10 \mathrm{~s}$. Operating on harvested energy poses different challenges. For one such system, prolonged $(10+s)$ full power operation is impossible because only the energy storage in capacitor banks is available. Unlike battery powered devices, this system is unable to power the Zigbee protocol tasks for prolonged periods of time. To prevent uncontrolled device power down, a return to sleep mode is mandatory within a time frame, as illustrated in Fig. 9b. Energy harvesting for WSN requires a meaningful change in node behavior when compared to battery operated nodes. Nodes running only on capacitor charge must be programmed so that short term energy constraints are met while battery operated nodes have strong long term energy limitations. In this system, short time energy availability is small. On the other hand, in the long run, energy is harvested allowing long term device powering. In this paper we propose to change the original Z-Stack implementation so that a full Zigbee compliant node is possible to run on harvested power. 


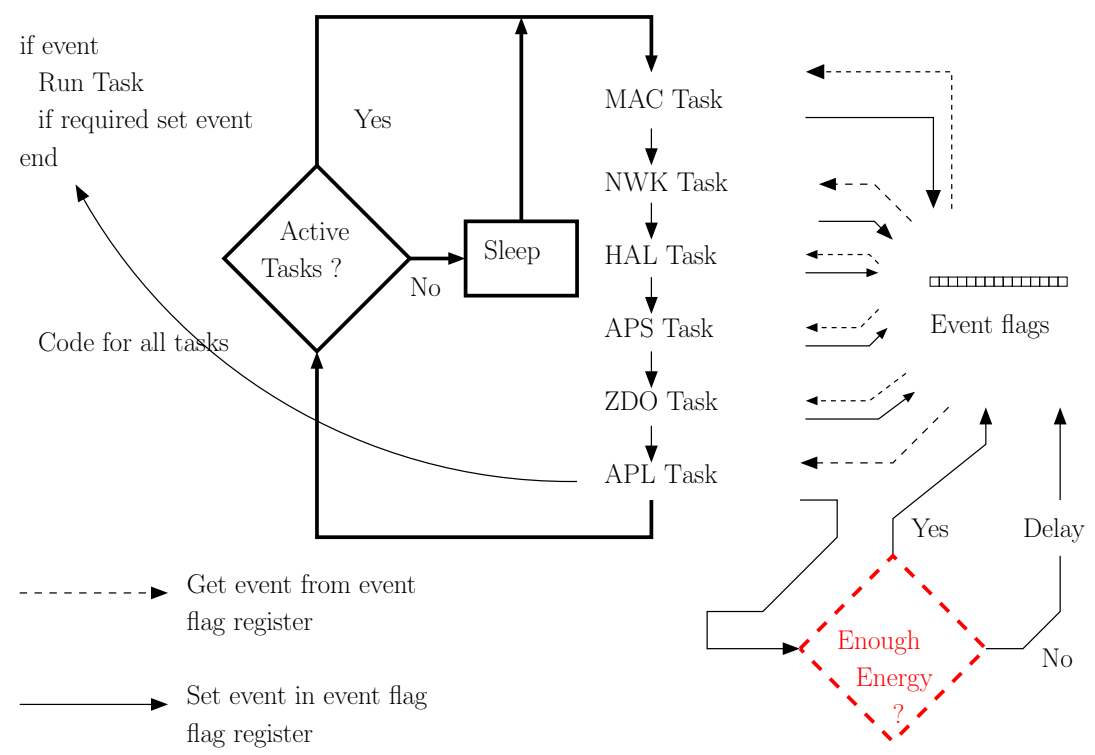

Fig. 12. Z-Stack OSAL task scheduling mechanism for an example task array. The OSAL reads the event flag register to determine whether a task should be launched. The event flag register is set by each task if required. The processor is allowed to sleep if no tasks have pending events. Application level tasks prevent event generation if no energy is available.

Figs. 11 and 12 illustrate original and proposed OSAL operation, signaling proposed changes with the dotted/ red $^{2}$ flowchart boxes. The software executes system initialization (see Fig. 11) before entering into the stack main loop (see Fig. 12). Changes are proposed to the initialization procedure as well as to the main round-robin operation. The system initialization executes start-up to memory allocation system, OSAL basic timer, message queue, power management and task scheduler system. With the proposed mechanism, tasks are executed only if enough energy is available on $V_{\text {main }}$ capacitor and the capacitor voltage level is used to determine operation capability. Fig. 11 illustrates the initialization procedure where energy availability is tested before system flash initialization because these memory accesses are, together with radio module operation, the CC2530 main current sinks. If the illustrated test returns a valid voltage level for $V_{\text {main }}$ capacitor the system is allowed to start OSAL operation. In OSAL, application processes run based on tasks that must be created in software. This is accomplished by creating an array of tasks and adding entries for each new application process. The OSAL searches for pending events created by tasks and only after they all agree the processor is allowed to sleep. As task execution is done by OSAL without user interference, the task scheduling events must be controlled to prevent processes from running. A typical operation sequence is initiated by an event set by hardware or by the application related tasks. These tasks implement the system main purpose of transferring user data with a defined periodicity reacting also to hardware changes. The remaining tasks get events that have been created by these parent tasks. Moreover, tasks that entail large energy costs are related with data transfers that

\footnotetext{
${ }^{2}$ For interpretation of color in Figs. 11 and 12, the reader is referred to the web version of this article.
}

are all application defined. Users must know at programming time the worst case sink current for each scheduled task. Application level tasks are allowed to schedule events only if energy availability exists. If a communication event is not possible, a delay event is set, thus allowing OSAL to place the processor in low power mode. Fig. 12 illustrates the round-robin task servicing scheme, the event scheduling process and the proposed blocking mechanism. Each task searches the event flag register to determine if an event has been generated. If an event is detected the task runs to completion and generates its own events if so required. Further transactions between tasks can be addressed using the messaging scheme also implemented in Z-Stack. Fig. 12 shows the implemented task array with Z-Stack. The stack has been configured so that all events are generated by the application defined task and hardware events disabled. The implemented mechanism operates therefore by blocking application initiated task events. The analysis of the implemented algorithm is presented in the next section.

\section{IEEE802.15.4/Zigbee task scheduler algorithm analysis}

The previous section described the proposed mechanism for task scheduling of ZED operation on capacitor stored energy. Since the main design constraint is the microcontroller voltage line level, the task scheduler objective is to maintain its value within operational limits.

\subsection{Model description and assumptions}

The proposed mechanism operation may be characterized by two operation modes: 
Before network join - In this mode at least one Active Scan is required for operation. Because of its impact on $V_{\text {main }}$, Active Scan scheduling is a simple mater of allowing it or not. No parameters are required to evaluate if this action is executed. Active Scan and Join actions are only executed with $V_{\text {main }}$ full charge. Moreover, these actions are usually associated with non periodic events. The proposed system has been designed so that capacitors are able to power three Active Scan procedures, as illustrated in Fig. 4.

After network join - In this mode, Zigbee data transfers entail a relative small impact on $V_{\text {main }}$ voltage level when compared to Active Scan/Join procedures. This mode allows data transfer management to be executed with a number of operational options, namely related with communication periodicity, data size and CSMA/ CA parameters. The task scheduler decision generates a new event, based on the possibility of a node power loss.

The event scheduler management operation must know beforehand the worst case scenario of a new task generated sink current. System parameters can be defined at hardware level, software compilation time and run time. The scheduler must also account for external parameters. The capacitance value is defined at hardware level and is therefore out of the scheduler control. In this system, the mains current is the external parameter. The compile time parameters are:

Worst case CSMA/CA - The parameters aMinBE, aMaxBE, aMaxBO as well as aMaxFR (described in sequel), represent the worst case communication time with the RF module turned on. These parameters cannot be changed during runtime. The scheduler must therefore account for their effects in current consumption, always assuming that maximum values are reached.

Number of bytes - The number of transferred bytes can be regarded as a compile time parameter. If the node is to maintain Zigbee Cluster Library [65] compatibility it is reasonable to assume that the clusters have fixed length payloads. Data transfers are achieved by Endpoint matching between two nodes. This matching is implemented by defining on both sender and destination nodes a common data structure addressed by a simple descriptor [47]. The contents of this data structure are transferred along with its identification that includes Endpoint number and profile identification. The architecture of the simple descriptor allows all possible data lengths in bits, thus enabling all sorts of data transfers. The fact that this structure must be known by both source and destination nodes, implies that for each particular case the data size parameter is known at compilation time.

Network message delivery timeouts - In a Zigbee network with sleeping end devices, data delivery is dependent on ZED poll rate and the parent defined indirect message relaying timeout. The indirect message timeout parameter should be defined so that messages are not discarded before the ZED defined poll rate. In ZStack, the indirect message timeout is a parent compile time defined parameter that the network must be aware.

The communication/poll period is a run time parameter and is therefore under the scheduler full control. Moreover, the scheduler must decide if the node is running out of energy and issue a network leave command thus announcing its absence to the network.

A model is proposed that addresses all mentioned parameters, allowing the task scheduler to decide if a communication event can be generated. The task scheduler manages the energy balance between the node consumption, and the harvested and capacitor storage energies. Eq. (4) describes this required energy balance.

$E_{\text {Cmain }}=-E_{\text {Active }}-E_{\text {sleep }}+E_{\text {harvesting }}$

where $E_{\text {Cmain }}$ is the capacitor $V_{\text {main }}$ available energy, $E_{\text {Active }}$ is the energy consumed by the node when transmitting, receiving or processing data, $E_{\text {sleep }}$ is the energy consumed by the node when in power down mode and $E_{\text {harvesting }}$ is the energy supplied by the SCCT harvester. The mechanisms by which the system consumes energy were described in Sections 4 and 3.3. Sleep mode energy consumption is also accounted for as constant. Note that the sleep energy includes capacitor self-discharge current as well as the CC2530 current in low power mode. The total value for the sleep current is considered to be $21 \mu \mathrm{A}$, where $20 \mu \mathrm{A}$ is accounted for the Cellergy capacitor [60] and $1 \mu \mathrm{A}$ for CC2530 [51] Power Mode 2 in which only Sleep Timer is running. The node active power consumption time has variable length and is highly dependent on communication environment. Understanding worst case active mode current consumption is required to determine $E_{\text {Active }}$ value. Active time can be described by [52]

$t_{\text {Active }}(n)=t_{s w t}+t_{T x R x}(n)+t_{c s m a}+t_{a c k}$

where $t_{\text {Active }}$ is the total time the node is transmitting or receiving $n$ bytes, $t_{a c k}$ is the acknowledgment procedure time, $t_{s w t}$ is the radio module receive/transmit or transmit/receive switching time, $t_{T X R X}(n)$ is the communication time and $t_{\text {csma }}$ is the CSMA/CA algorithm running time. In the case of $t_{a c k}$, transmission is executed without CSMA/ CA mechanism thus entailing a fixed time length (see Section 3.3).

The CSMA/CA mechanism is used to avoid communication collision and energy consumption in noisy RF environments. As described in Section 3.3, nodes execute carrier sensing to assess radio channel availability based on a randomly distributed fixed length medium sampling. CSMA/ $\mathrm{CA}$ is not however fail proof and collisions occur. In this case, when CSMA/CA informs upper layers that the radio channel is clear to send, a communication is attempted. If other node is also communicating or the radio channel is otherwise occupied, data transfer fails and the destination node does not send MAC level acknowledgment. A node that fails to receive data acknowledgment may retry communication. From (2) the maximum time a node tries to send data can be written as

$t_{\text {TxRxmax }}(n)=(\operatorname{aMaxFR}+1) \cdot t_{T x R x}(n)$ 


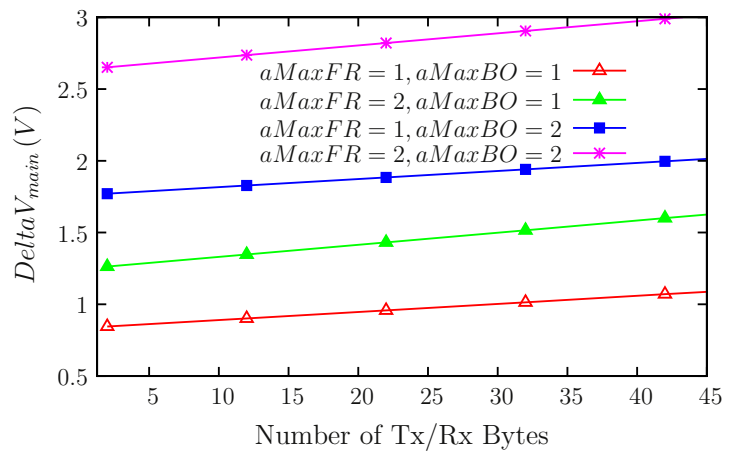

(a)



(b)

Fig. 13. $V_{\text {main }}$ voltage drop for $n$ bytes communication as a funtion of Frame retries and backoff periods for mains current of 1.2 A. (a) The $V_{\text {main }}$ capacitor has $330 \mu \mathrm{F}$. (b) The $V_{\text {main }}$ capacitor has $470 \mu \mathrm{F}$.

where $a M a x F R$ is the maximum number of transmit retries (frame retries) a node executes on each communication attempt. The number of frame retries in both Z-Stack and 802.15.4 definition is set by default to 3. As communication retries require a new CSMA/CA procedure execution, from (1) the maximum time for the CSMA/CA mechanism $t_{\text {CSMAmax }}$ may be written as

$t_{\text {CSMAmax }}=(\operatorname{aMaxFR}+1) \cdot t_{\text {CSMA }}$

As shown in Section 3.3, the CSMA/CA listening time expressed in (1), is dependent on software parameter values $a M i n B E, a M a x B E$ and $a M a x B O$ that are respectively set by default to 3, 5 and 4 in both IEEE802.15.4 definition and Z-Stack implementation. The unit backoff period $t_{\text {aUnitBo }}$ of (1) is $320 \mu \mathrm{s}$ for $2.4 \mathrm{GHz}$ frequency band. Also in (5), $t_{s w t}, t_{a c k}$ entail constant periods of time with $192 \mu \mathrm{s}$ and $864 \mu \mathrm{s}$, respectively. Harvested energy is dependent on mains current behavior.

The energy balance in (4) can be described as a function of the $V_{\text {main }}$ capacitor voltage as:

$$
\begin{aligned}
V_{C}(T)-V_{C}(0)= & \frac{1}{C} \\
& \cdot \int_{0}^{T}\left[-i_{\text {active }}(t, n)-i_{\text {sleep }}(t)+i_{\text {harvested }}(t)\right] d t
\end{aligned}
$$

where $V_{C}(T)$ is the $V_{\text {main }}$ capacitor voltage after a charge/ discharge period $T$ measured between two consecutive data transfers, $V_{C}(0)$ is the initial $V_{\text {main }}$ capacitor voltage, $i_{\text {active }}(t, n)$ is the node instantaneous active current to transfer $n$ bytes at time instant $t, i_{\text {sleep }}$ is the node instantaneous current when in sleep mode, and $i_{\text {harvested }}$ is the instantaneous current the AC/DC circuit is able to harvest.

Eq. (8) can be written as

$$
\begin{aligned}
\Delta V_{\text {Cmain }}= & \frac{I_{\text {Active }}(n)}{C} \cdot t_{\text {active }}+\frac{I_{\text {Sleep }}}{C} \cdot\left(T-t_{\text {active }}\right) \\
& -\frac{I_{\text {harvested }}}{C} \cdot T
\end{aligned}
$$

where $\Delta V_{\text {Cmain }}$ is the voltage variation of $V_{\text {main }}$ when an $I_{\text {Active }}$ current is sinked during a $t_{\text {active }}$ time within a period $T$, with $n$ transferred bytes. $I_{\text {sleep }}$ is the capacitors' self dis- charge current plus the CC2530 power down current and $I_{\text {harvested }}$ is the harvested current for time interval $T$, where $T>t_{\text {active }}$. The model addresses harvested current with a discrete behavior $" I_{\text {harvested }}=\{700 \mathrm{~mA}, 800 \mathrm{~mA}, 900 \mathrm{~mA}$, $1 \mathrm{~A}, 1.1 \mathrm{~A}, \ldots, 2 \mathrm{~A}, 3 \mathrm{~A}, 4 \mathrm{~A}, \ldots, 10 \mathrm{~A}\}$. Current $I_{\text {active }}$ is the average piecewise value of $i_{\text {active }}$ during the time interval of (5).

\subsection{Model analysis}

The $V_{\text {main }}$ voltage level is the main constraint the operational mechanism must address. Fig. 13 illustrates $V_{\text {main }}$ voltage drop for $n$ bytes data transfers for different values of frame retries and for a number of backoff periods with $470 \mu \mathrm{F}$ and $330 \mu \mathrm{F}$ capacitors. These capacitor values have been used in the system proposed in [57]. For the proposed device, the maximum $V_{\text {main }}$ supported variation is $1.5 \mathrm{~V}$. Fig. 13 shows that $330 \mu \mathrm{F}$ capacitor values can only operate with $a M a x B O$ value of 1 and 10 transferred bytes. A different number of maximum backoff procedures entails a $V_{\text {main }}$ variation larger than the supported by the capacitor charge. For a $470 \mu \mathrm{F}$, a $V_{\text {main }}$ drop outside device operational limits is achieved if aMaxBO and aMaxFR are both 2 . For these capacitor values the system has limited operational capabilities but can be used with the scenario proposed in [41]. In this case 10 bytes are shown to be enough to monitor induction motor parameters. Also the nodes are placed after network formation which is allowed by the contactless architecture. Fig. 14 shows system operation with $V_{\text {main }}$ Cellergy available capacitor series of $240 \mathrm{mF}$ and $160 \mathrm{mF}$. The effect of these capacitors is clearly shown by the fact that $V_{\text {main }}$ voltage variation is measured in millivolt. With this hardware configuration the device is able to power the Out-of-the-Box Z-Stack with payload length illustrated up to 45 bytes. The system is able to sustain the maximum number of frame and backoff retries defined at compile time with the default Z-Stack distribution. Figs. 13 and 14 illustrate system behavior for the two implemented hardware versions. In Fig. 15, the backoff exponent (aMaxBE) impact on the $V_{\text {main }}$ voltage is addressed. This analysis is done for the worst case scenario in which the backoff exponent is always accounted for its 


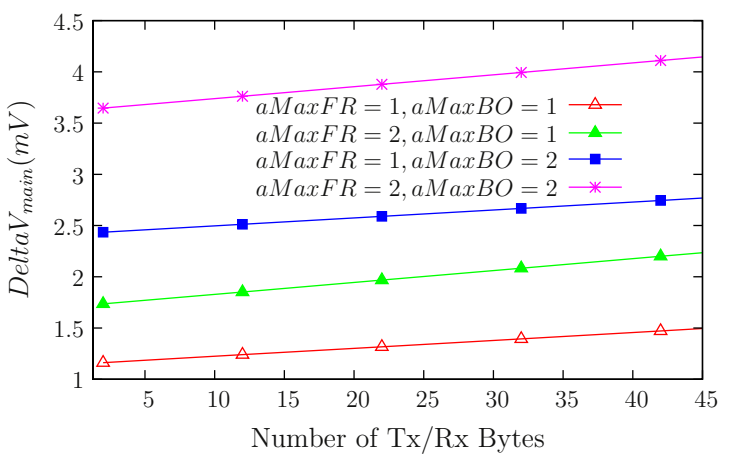

(a)

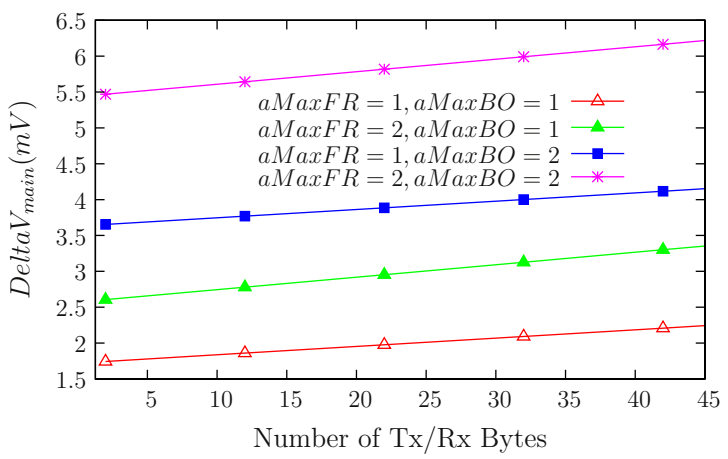

(b)

Fig. 14. $V_{\text {main }}$ voltage drop for $n$ bytes communication as a function of Frame retries and backoff periods for mains current of 1.2 A. (a) The $V_{\text {main }}$ capacitor has $240 \mathrm{mF}$. (b) The $V_{\text {main }}$ capacitor has $160 \mathrm{mF}$.

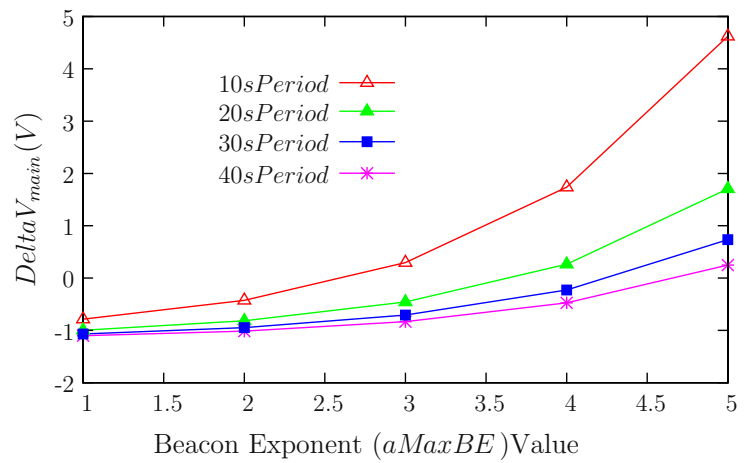

(a)

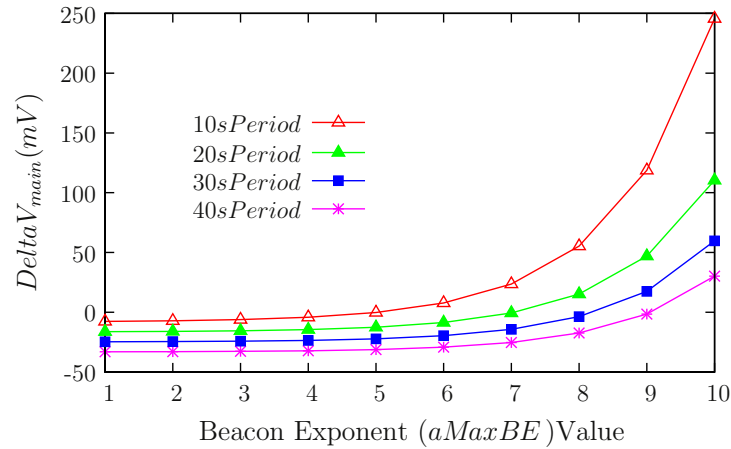

(b)

Fig. 15. $V_{\text {main }}$ voltage drop for the worst case bakoff exponent, defined by aMinBE and $a M a x B E$, as a function of the data transfer period for mains current of 1.2 A. Also aMaxBO is set to 3, aMaxFR is set to 2 and 50 bytes are transferred. (a) The $V_{\text {main }}$ capacitor has $330 \mu \mathrm{F}$. (b) The $V_{\text {main }}$ capacitor has $240 \mathrm{mF}$.

maximum value. The backoff exponent is shown to have impact on system sustainability if a small data transfer period is implemented. For a $330 \mu \mathrm{F}$ capacitor, a backoff exponent parameter larger than 3 and a periodic data transfer of $10 \mathrm{~s}$ entails a $V_{\text {main }}$ voltage drop outside the device operational limits. For this capacitor values, larger periods may be used as $V_{\text {main }}$ voltage drop is sustainable up to a value of 5 of the $a M a x B E$ parameter. The $V_{\text {main }}$ voltage variation measured in millivolt shows the relatively small impact suffered by the $240 \mathrm{mF}$ capacitor to the $a M a-$ $x B E$ parameter variation. The analysis for a different hardware configuration (e.g. different capacitor values) is illustrated in Fig. 16. This analysis shows how changing $V_{\text {main }}$ capacitor values allows system communication with different period $T$. A $\Delta V_{\text {main }}$ value of zero shows that an energy balance exists between the harvested and the drawn energy. Negative values stand for a surplus of harvested energy as a positive variation illustrates that the node is consuming more energy than the one it scavenges. Moreover, a limit to the harvested energy is defined within the model so that real capacitor storage is accounted for. The limit to the harvested energy is illustrated by the minimum negative $\Delta V_{\text {main }}$ which means that the capacitor has reached charge limit and thus no more energy can be stored. Fig. 16 also illustrates that smaller capacitor values charge faster, by reaching maximum stored energy, and can therefore be better suited to enhance system availability and smaller communications period. Fig. 17 shows model behavior for periodic communications. It is shown that the number of bytes has little impact on $V_{\text {main }}$ varia-

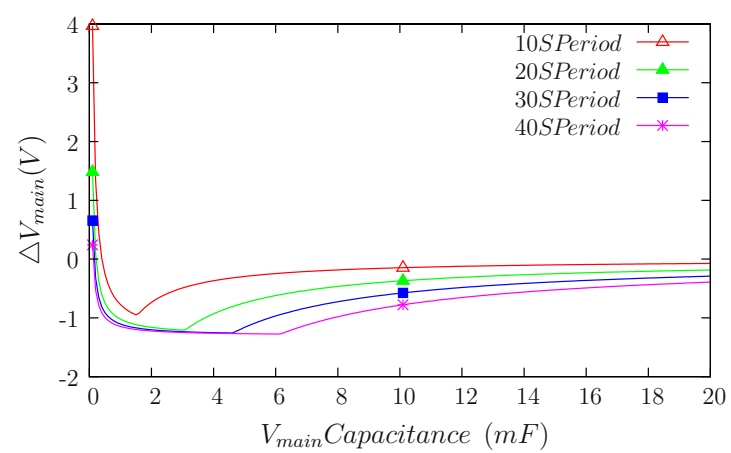

Fig. 16. $V_{\text {main }}$ voltage variation analysis for capacitance value and period T. Mains current is accounted as $1.2 \mathrm{~A}$ and MAC parameters as their default. 


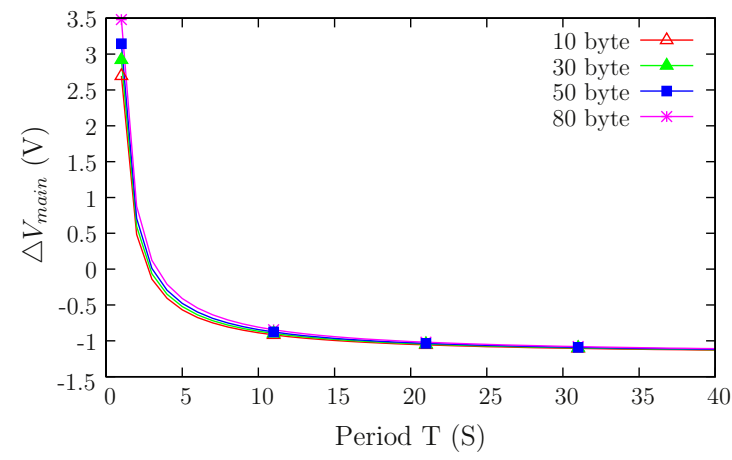

(a) $V_{\text {main }}$ capacitande $330 \mu F$

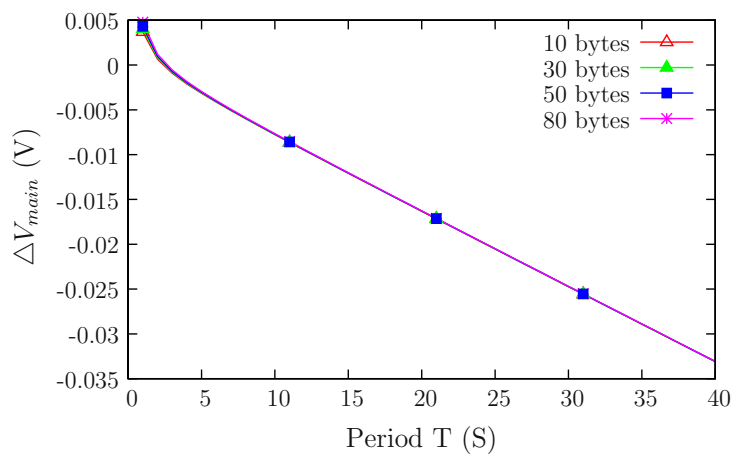

(b) $V_{\text {main }}$ capacitande $240 \mathrm{mF}$

Fig. 17. $V_{\text {main }}$ voltage variation analysis for communication period $(\mathrm{T})$ and number of transferred bytes. Mains current is accounted as $1.2 \mathrm{~A}$ and $\mathrm{MAC}$ parameters as their default.

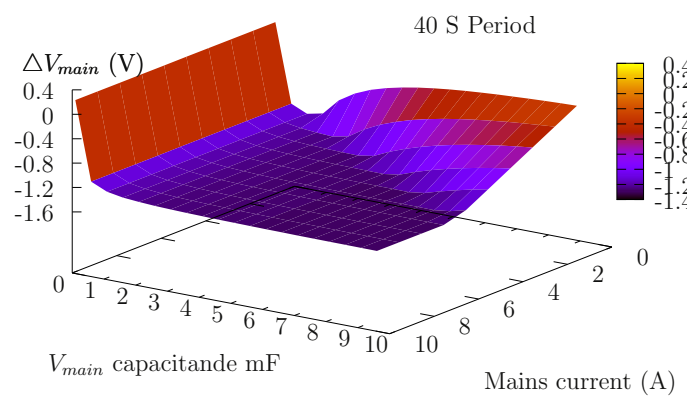

(a) $V_{\text {main }}$ capacitande $330 \mu \mathrm{F}$



40 S Period

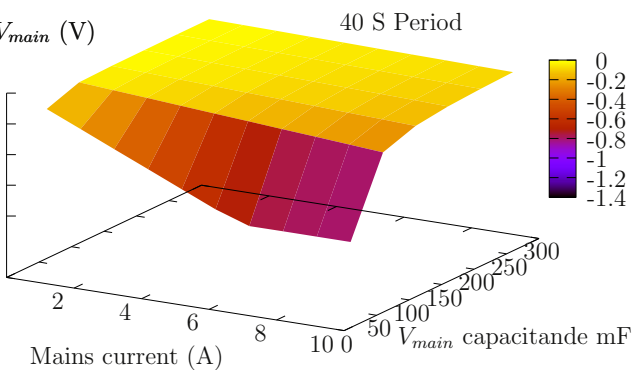

(b) $V_{\text {main }}$ capacitande $240 \mathrm{mF}$

Fig. 18. $V_{\text {main }}$ voltage variation analysis for of mains current and $V_{\text {main }}$ capacitante. Communication period (T) of $40 \mathrm{~s}$, 40 transferred bytes and MAC parameters default values.

tion. Fig. 17a is consistent with the measurements presented in Section 4 where 5 -s spaced communications are compatible with capacitor recharging as for this communication period a negative voltage variation is illustrated. Moreover, Fig. 17b illustrates that large capacitors present small $V_{\text {main }}$ variations as well as large recharge times represented by the fact that no asymptote exists for these curves. Fig. 18 shows the model analysis of the mains current and the $V_{\text {main }}$ capacitance for a 40 -s communication period. Fig. 18a represents model behavior for $\mu \mathrm{F}$ range capacitance values and Fig. $18 \mathrm{~b}$ shows results for $\mathrm{mF}$ range capacitors.

\subsection{Model applications and mechanism compliance}

The proposed device is to be included in a Zigbee network as a ZED. As all defined ZEDs in Zigbee, it will not execute routing tasks nor data relay. The proposed device must however be able to receive data and implement all protocol functions mandated by the Zigbee definition. ZED operation requires that the device is able to respond to mandatory services. These services are provided by the Zigbee Device Profile and are associated with discover, maintain and configure the network. A client-server mechanism is implemented in which server side services are mandatory. All devices must respond with their short and long addresses and their node and power descriptors when requested, thus exposing device level characteristics. Moreover implemented services (Endpoints) must also be exposed when requested by some node with authority. In this case simple descriptors and active Endpoints are transmitted when queried. Table 2 shows the Zigbee services with mandatory response by all network nodes. The number of bytes required by each service is identified in this table as well as the type of service and which node is the responder. For sleeping nodes the parent node can assume the child's role and respond on its behalf. Table 2 shows that some services exceed the 10 byte communication limit determined in the previous section for the device with the $\mu \mathrm{F}$ range capacitors. The considerations of the previous section have been obtained for the worst case scenario in which the $\mathrm{MaCHr}$ (see Section 3.1) is computed for a value of 20 bytes. The implemented $\mu \mathrm{F}$ device uses a $\mathrm{MacHr}$ of 6 bytes thus allowing it to support the mandatory services payload. The $\mathrm{mF}$ range capacitor devices have been shown to suffer little influence from the number of transferred bytes.

In a Zigbee network with sleeping end devices, ZED data delivery is dependent on its poll rate and the parent indirect message relaying timeout defined for router devices. The Zigbee Alliance defines Protocol Implementation Conformance Statement (PICS) to specific device profiles. PICS 


\section{Table 2}

Implemented device mandatory services with number of bytes, service type and responder in case of non powered devices.

\begin{tabular}{llll}
\hline Service & $\begin{array}{l}\text { Number of } \\
\text { bytes }\end{array}$ & Type & Responder \\
\hline Network address & 12 & Device discovery & Parent \\
IEEE address & 12 & Device discovery & Node \\
Node descriptor & 17 & Device discovery & Node \\
Power descriptor & 6 & Device discovery & Node \\
Device anounce & 12 & Device discovery & Parent \\
Simple descriptor & 15 & Service discovery & Node \\
Active end point & 8 & Service discovery & Node \\
Match descriptor & 6 & Service discovery & Node \\
\hline
\end{tabular}

classifies parameters and functionalities for a determined profile. The poll rate and the indirect message timeout are two of these PICS defined parameters. As an example the Zigbee specification recommends polling at least once per $7.5 \mathrm{~s}$ but the Smart Energy profile allows this poll rate latency to be as large as $60 \mathrm{~s}$. In either case the indirect message timeout parameter should be defined so that messages are not discarded before the ZED defined poll rate. The proposed system relies on the mains current availability and on its absence the node energy source is unable to power the node. Moreover, on the router side, message relay is a memory consuming procedure that can be affected by ZED unavailability. An absent ZED is unable to poll its master and may therefore be discarded and removed from the controller association table.

A six node network experimental setup has been implemented in which one node is configured as a battery powered ZED, one node is configured as a SCCT powered ZED, three nodes are routers and a central sink node is the coordinator. All nodes implement a time synchronization service in which the coordinator broadcasts its date/time in UTC (Coordinated Universal Time) format, entailing a periodic 4 byte data broadcast. Routers receive the UTC time for themselves and relay it for their children if any. Both ZED transfer to the sink node, in unicast mode, a 16 byte data packet with their $V_{\text {main }}$ and $V_{\text {storage }}$ values ( 2 bytes each), synchronized UTC time (4 bytes), short address ( 4 bytes), 2 bytes with a communication index and 2 bytes with data tags for parsing at the sink. This data packet is sent with adjustable periodicity that may be changed from any other node within the network in unicast mode. The period change command is implemented with a 2 bytes structure. Each node is therefore implemented with three Endpoints. Fig. 19 illustrates $V_{\text {main }}, V_{\text {storage }}$ variation with time and different mains currents for the SCCT powered ZED. The proposed mechanism effect is also shown by changing the data transfer periodicity. The capacitor is initially fully charged at 13:30:00 h. A 5-s data transfer periodicity is thereafter initiated with the $V_{\text {main }}$ and $V_{\text {storage }}$ readings. Mains current behavior is also shown. In this experimental setup the measurements are obtained by the node resulting that no data is available on a power failure. The proposed mechanism operates therefore by increasing the period of readings thus signaling a low voltage at $V_{\text {main }}$. Initial period communication is restored as $V_{\text {main }}$ recharges over the mechanism activation threshold. In [66] a study is proposed on the existing definitions of network lifetime. Also a generic definition of network lifetime is proposed. A number of lifetime definitions are based on network connectivity metrics. Connectivity is defined as the ability to transmit data to a particular destination. In our system this ability can be defined as the time a node remains operational in the absence of mains current. Also the proposed device assumes the remaining network is fully operational and therefore able to relay data to a particular destination. The node availability is therefore discussed as the capability to poll data within the limits tolerated by the Zigbee definition. Figs. 20 and 21 show measured best and worst case for device lifetime with the proposed mechanism. The measurements have been obtained with no mains current and communication period of $60 \mathrm{~s}$ as the best case scenario. The worst case scenario entails a $1 \mathrm{~s}$ period communications. For the $60 \mathrm{~s}$ period, shown in Fig. 20, the device is able to comply with the Zigbee protocol for 04:30 h without mains current energy supply. After this period the proposed mechanism

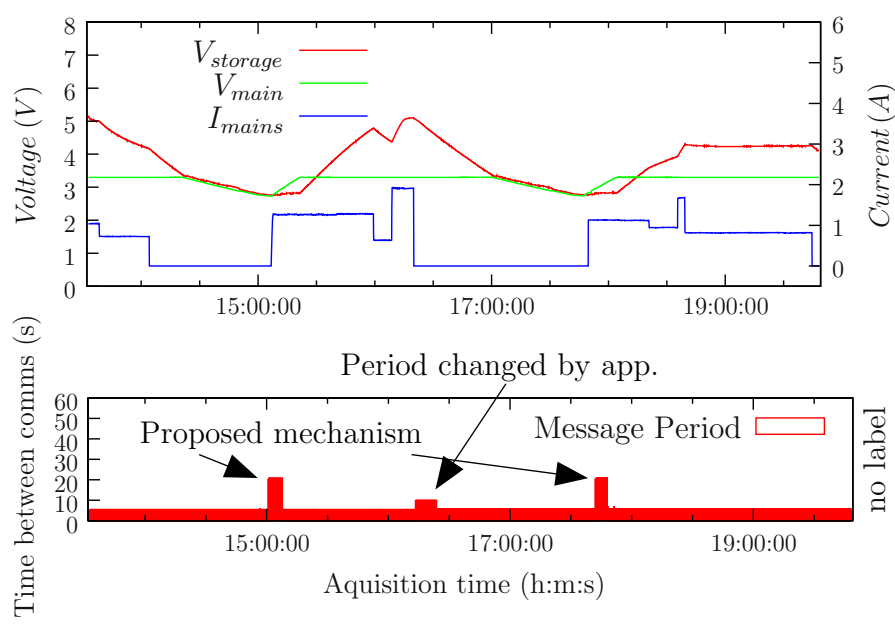

Fig. 19. $V_{\text {main }}, V_{\text {storage }}$ voltage, $I_{\text {mains }}$ (upper graph) and time between two data transfers (lower graph) illustrating capacitor charging with mains current. The proposed mechanism operation is identified by a change in the data transfer period when $V_{\text {main }}$ reaches a low charge value. Also the transmission period changed by the network application software is shown. 


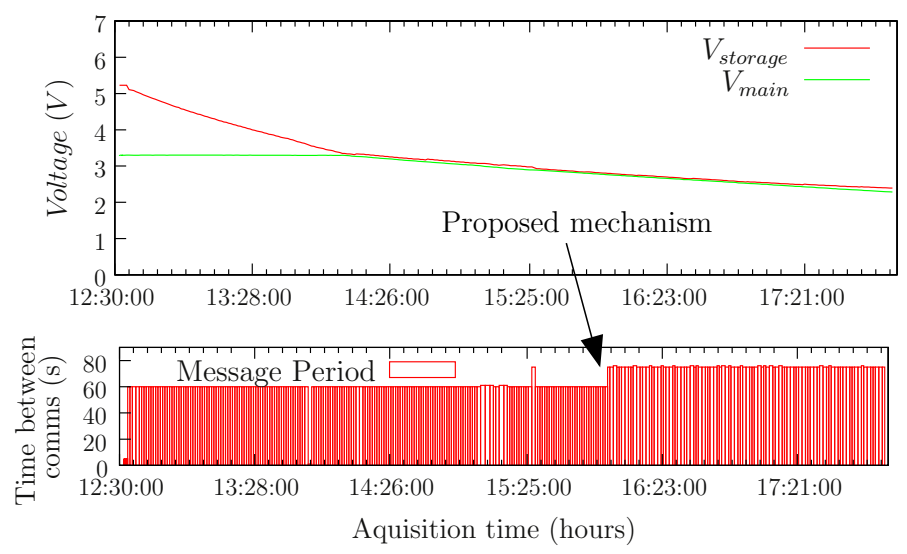

Fig. 20. Device lifetime for best case communication period of $60 \mathrm{~s}$.



Fig. 21. Device lifetime for worst case communication period of $1 \mathrm{~s}$.

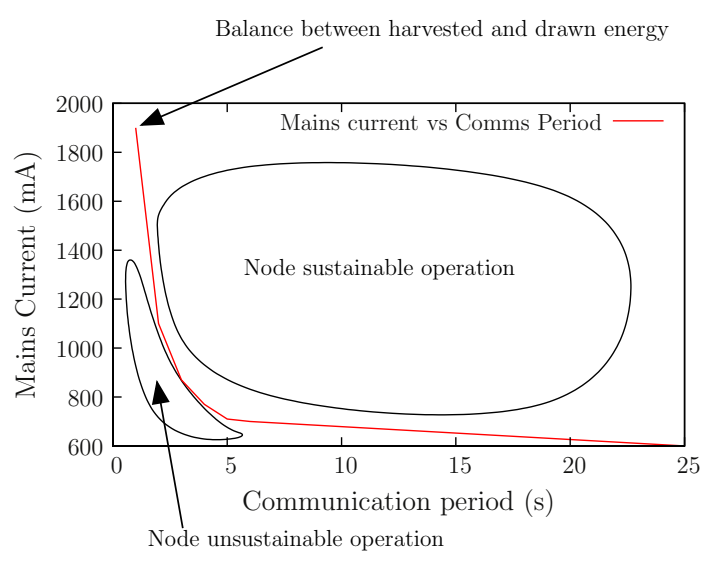

Fig. 22. Communications period balance with mains current delimiting sustainable device operation.

is activated and two more operational hours are possible with communication periodicity of $70 \mathrm{~s}$. In the worst case scenario, shown in Fig. 21, the device is powered for 01:45 $\mathrm{h}$ and for one more hour with the activated mechanism. Fig. 22 illustrates mains current values that balance node energy consumption with periodic communications.
This figure shows a measure for device sustainability. Large mains currents allow the device to operate with higher communication frequency. For small mains current lower communication frequency is required.

\section{Conclusion}

Texas Instruments IEEE802.15.4/Zigbee protocol stack is a powerful implementation tool for WSN. It is nevertheless a complex implementation whose operation can be controlled through a large number of compile and run time options. Moreover the IEEE802.15.4/Zigbee specifications provides a strong support for battery operated nodes but does not consider nodes that run on harvested energy. The work presented in this paper addresses this omitted characteristic of IEEE802.15.4/Zigbee definition. The proposed device is able to operate within a Zigbee network, maintaining network connectivity and data delivery rate. The device responds to Zigbee mandatory services and is remotely configurable by other network nodes. Moreover, a time synchronization service is implemented within the network including the proposed device. Within the context of battery-free nodes, IEEE802.15.4/Zigbee Active Scan and Join activities are found to be the critical ones. In this work, an energy management strategy is implemented based on 
available tools. The proposed solution uses Texas Instruments Z-Stack Zigbee software bundle. The original Texas Instruments software stack is successfully adapted to operate on harvested energy. Performed adaptation allowed IEEE802.15.4/Zigbee protocol compliance to be maintained while system operation is made possible for a micro power scavenger. Our harvester device can be used in multiple industrial and civil applications, such as large building energy monitoring and industrial devices monitoring. Also the device can be used for educational purposes to simulate WSN node operation running on harvested energy. The operation on ATEX (Explosive Atmospheres ${ }^{3}$ ) environments is a possible use of the proposed device because of its small charge accumulation and contact-less architecture. Further improvements can be added to the mechanism. The proposed mechanism operation relies on $V_{\text {main }}$ analysis to determine device status. On $V_{\text {main }}$ full charge the device operates as a battery powered node. $V_{\text {storage }}$ analysis can therefore be included in the proposed mechanism.

\section{Acknowledgment}

This work was partially supported by FCT (Portuguese Science and Technology Foundation): PHD Grant SFRH/ $\mathrm{BD} / 45602 / 2008$.

\section{References}

[1] I.F. Akyildiz, T. Melodia, K.R. Chowdhury, A survey on wireless multimedia sensor networks, Comput. Networks 51 (4) (2007) 921960.

[2] L. Atzori, A. Iera, G. Morabito, From smart objects to social objects: the next evolutionary step of the internet of things, Commun. Mag., IEEE 52 (1) (2014) 97-105, http://dx.doi.org/10.1109/MCOM.2014. 6710070.

[3] F. Ferreira, A. de Almeida, Novel multiflux level, three-phase, squirrel-cage induction motor for efficiency and power factor maximization, IEEE Trans. Energy Convers. 23 (1) (2008) 101-109.

[4] S. Chalasani, J.M. Conrad, A survey of energy harvesting sources for embedded systems, in: Southeastcon, IEEE, 2008, pp. 442-447.

[5] S. Sudevalayam, P. Kulkarni, Energy harvesting sensor nodes: survey and implications, Commun. Surv. Tutor., IEEE PP (99) (2010) 1-19.

[6] G. Tewolde, Current trends in low-power embedded computing, in: 2010 IEEE International Conference on Electro/Information Technology (EIT), 2010, pp. 1-6. http://dx.doi.org/10.1109/EIT. 2010.5612129.

[7] S. Beeby, N. White, Energy Harvesting for Autonomous Systems, Artech House, 2010.

[8] A. Harb, Energy Harvesting: State-of-the-art, vol. 36, Elsevier, 2011.

[9] R. Freeland, Energy harvesting: a practical reality for wireless sensing, 2012 <http://www.perpetuum.com/resources.asp>.

[10] R. Vullers, R. van Schaijk, I. Doms, C.V. Hoof, R. Mertens, Micropower energy harvesting, Solid-State Electron. 53 (7) (2009) 684-693.

[11] M. Kuorilehto, M. Kohvakka, J. Suhonen, P. Hamalainen, M Hannikainen, T.D. Hamalainen, Ultra-Low Energy Wireless Sensor Networks in Practice: Theory, Realization and Deployment, Wiley Publishing, 2008.

[12] W. Seah, Z.A. Eu, H.P. Tan, Wireless sensor networks powered by ambient energy harvesting (wsn-heap) - survey and challenges, in: 1st International Conference on Wireless Communication, Vehicular Technology, Information Theory and Aerospace Electronic Systems Technology, 2009, Wireless VITAE 2009, 2009, pp. 1-5.

[13] C. Carvalho, J. Oliveira, N. Paulino, Survey and analysis of the design issues of a low cost micro power dc-dc step up converter for indoor light energy harvesting applications, in: 2012 Proceedings of the 19th International Conference Mixed Design of Integrated Circuits and Systems (MIXDES), 2012, pp. 455-460.
[14] A. Weddell, G.V. Merrett, B. Al-Hashimi, Photovoltaic sample-andhold circuit enabling mppt indoors for low-power systems, IEEE Trans. Circ. Syst. I: Regular Papers 59 (6) (2012) 1196-1204, http:// dx.doi.org/10.1109/TCSI.2011.2173393.

[15] A. Decker, Solar energy harvesting for autonomous field devices, Wireless Sensor Systems, IET 4 (1) (2014) 1-8, http://dx.doi.org/ 10.1049/iet-Wss.2013.0011.

[16] S. Ghosh, H.T. Wang, W. Leon-Salas, A circuit for energy harvesting using on-chip solar cells, IEEE Trans. Power Electron. 29 (9) (2014) 4658-4671, http://dx.doi.org/10.1109/TPEL.2013.2273674.

[17] W. Chye, Z. Dahari, O. Sidek, M. Miskam, Electromagnetic micro power generator; a comprehensive survey, in: 2010 IEEE Symposium on Industrial Electronics Applications (ISIEA), 2010, pp. 376-382.

[18] K. Tao, G. Ding, P. Wang, Z. Yang, Y. Wang, Fully integrated micro electromagnetic vibration energy harvesters with micro-patterning of bonded magnets, in: 2012 IEEE 25th International Conference on Micro Electro Mechanical Systems (MEMS), 2012, pp. 1237-1240. http://dx.doi.org/10.1109/MEMSYS.2012.6170413.

[19] K. Ono, N. Sato, T. Shimamura, M. Ugajin, T. Sakata, S. Mutoh, et al., A millimeter-sized electret-energy-harvester with microfabricated horizontal arrays and vertical protrusions for power generation enhancement, in: 2011 16th International, Solid-State Sensors, Actuators and Microsystems Conference (TRANSDUCERS), 2011, pp. 1863-1866. http://dx.doi.org/10.1109/TRANSDUCERS.2011. 5969846.

[20] X. Lu, S.H. Yang, Thermal energy harvesting for wsns, in: 2010 IEEE International Conference on Systems Man and Cybernetics (SMC), 2010, pp. 3045-3052.

[21] V. Leonov, Thermoelectric energy harvesting of human body heat for wearable sensors, Sensors J., IEEE 13 (6) (2013) 2284-2291, http:// dx.doi.org/10.1109/JSEN.2013.2252526.

[22] A. Post, C. Knight, E. Kisi, Thermomagnetic energy harvesting with first order phase change materials, J. Appl. Phys. 114 (3) (2013) 033915, http://dx.doi.org/10.1063/1.4815933.

[23] M. Kiziroglou, S. Wright, T. Toh, P. Mitcheson, T. Becker, E. Yeatman, Design and fabrication of heat storage thermoelectric harvesting devices, IEEE Trans. Ind. Electron. 61 (1) (2014) 302-309, http:// dx.doi.org/10.1109/TIE.2013.2257140.

[24] R. Vullers, R. Schaijk, H. Visser, J. Penders, C. Hoof, Energy harvesting for autonomous wireless sensor networks, Solid-State Circ. Mag., IEEE 2 (2) (2010) 29-38.

[25] Y. Ding, T. Arslan, A. Hamilton, Broadband antenna for rf energy scavenging system, in: Antennas and Propagation Conference (LAPC), 2012 Loughborough, 2012, pp. 1-4. http://dx.doi.org/ 10.1109/LAPC.2012.6403034.

[26] S.E. Adami, V. Marian, N. Degrenne, C. Vollaire, B. Allard, F. Costa, Self-powered ultra-low power dc-dc converter for rf energy harvesting, in: Faible Tension Faible Consommation (FTFC), 2012 IEEE, 2012, pp. 1-4. http://dx.doi.org/10.1109/FTFC.2012.6231746.

[27] R. Shigeta, T. Sasaki, D.M. Quan, Y. Kawahara, R. Vyas, M. Tentzeris, et al., Ambient rf energy harvesting sensor device with capacitorleakage-aware duty cycle control, Sensors J., IEEE 13 (8) (2013) 2973-2983, http://dx.doi.org/10.1109/JSEN.2013.2264931.

[28] B. Ulmen, P.D. Desai, S. Moghaddam, G.H. Miley, R.I. Masel, Development of diode junction nuclear battery using ${ }^{63} \mathrm{Ni}, \mathrm{J}$ Radioanal. Nucl. Chem. 282 (2) (2009) 601-604.

[29] A. Lal, R. Duggirala, H. Li, Pervasive power: a radioisotope-powered piezoelectric generator, IEEE Pervas. Comput. 4 (1) (2005) 53-61.

[30] D. yong Qiao, X.J. Chen, Y. Ren, W.Z. Yuan, A micro nuclear battery based on sic schottky barrier diode, J. Microelectromech. Syst. 20 (3) (2011) 685-690, http://dx.doi.org/10.1109/JMEMS.2011.2127448.

[31] H. Guo, Y. Shi, Y. Zhang, Y. Zhang, J. Han, Fabrication of sic p-i-n betavoltaic cell with 63ni irradiation source, in: 2011 International Conference of Electron Devices and Solid-State Circuits (EDSSC), 2011, pp. 1-2. http://dx.doi.org/10.1109/EDSSC.2011.6117636.

[32] N. Roscoe, M. Judd, Optimization of voltage doublers for energy harvesting applications, Sensors J., IEEE 13 (12) (2013) 4904-4911, http://dx.doi.org/10.1109/JSEN.2013.2278468.

[33] M. Zhu, P. Baker, N.M. Roscoe, M. Judd, J. Fitch, Alternative power sources for autonomous sensors in high voltage plant, in: 2009 IEEE Electrical Insulation Conference, 2009.

[34] T. Taithongchai, E. Leelarasmee, Adaptive electromagnetic energy harvesting circuit for wireless sensor application, in: 6th International Conference on Electrical Engineering/Electronics, Computer, Telecommunications and Information Technology, 2009, ECTI-CON 2009, vol. 01, 2009, pp. 278-281. http://dx.doi.org/ 10.1109/ECTICON.2009.5137008. 
[35] A. Kosonen, J. Ahola, Communication concept for sensors at an inverter-fed electric motor utilizing power-line communication and energy harvesting, IEEE Trans. Power Deliver. 25 (4) (2010) 24062413, http://dx.doi.org/10.1109/TPWRD.2010.2041374.

[36] H. Rashed, A. Bhuiyan Roger, M.A. Dougal, A miniature energy harvesting device for wireless sensors in electric power system, IEEE Sensors J. 10 (7) (2010) 1249-1258.

[37] D. Gunduz, K. Stamatiou, N. Michelusi, M. Zorzi, Designing intelligent energy harvesting communication systems, Commun. Mag., IEEE 52 (1) (2014) 210-216, http://dx.doi.org/10.1109/ MCOM.2014.6710085.

[38] N. Michelusi, K. Stamatiou, L. Badia, M. Zorzi, Operation policies for energy harvesting devices with imperfect state-of-charge knowledge, in: 2012 IEEE International Conference on Communications (ICC), 2012, pp. 5782-5787. http://dx.doi.org/ 10.1109/ICC.2012.6364958.

[39] R. Shigeta, T. Sasaki, D. Quan, Y. Kawahara, R. Vyas, M. Tentzeris, et al., Ambient RF energy harvesting sensor device with capacitorleakage-aware duty cycle control, Sensors J., IEEE 13 (8) (2013) 2973-2983.

[40] R. Moghe, A. Iyer, F. Lambert, D. Divan, A low-cost electric-field energy harvester for an mv/hv asset-monitoring smart-sensor, in: Energy Conversion Congress and Exposition (ECCE), 2013 IEEE, 2013, pp. 2676-2683. http://dx.doi.org/10.1109/ECCE.2013.6647047.

[41] J.P. Amaro, J. Ferreira, R. Cortesão, J. Landek, In-field operation monitoring of induction motors using wireless modules running on harvested power, in: Proceedings 37th Annual Conference of the IEEE Industrial Electronics Society, 2011, 2011a.

[42] Texas Instruments, Z-stack - zigbee protocol stack <http:// www.ti.com/tool/z-stack> (accessed Julye 2013a).

[43] R. Severino, M. Alves, A. Koubaa, On the Use of IEEE802.15.4/Zigbee for Time-Sensitive Wireless Sensor Network Applications, Master's thesis, ISEP, 2008

[44] Microchip Technology Inc., Zigbee pro stack <http:// www.microchip.com> (accessed June 2012).

[45] Z. Chen, C. Lin, H. Wen, H. Yin, An analytical model for evaluating IEEE 802.15.4 CSMA/CA protocol in low-rate wireless application, in: 21st International Conference on Advanced Information Networking and Applications Workshops, 2007, AINAW '07, vol. 2, 2007, pp. 899-904.

[46] T.R. Park, T. Kim, J. Choi, S. Choi, W. Kwon, Throughput and energy consumption analysis of IEEE 802.15.4 slotted csma/ca, Electron. Lett. 41 (18) (2005) 1017-1019.

[47] S. Farahani, ZigBee Wireless Networks and Transceivers, Newnes, Newton, MA, USA, 2008. ISBN 0750683937, 9780750683937.

[48] J.H. Lee, E.S. Lee, D.S. Kim, Network joining algorithm for mobile nodes in ubiquitous sensor networks, in: 2010 5th International Conference on Computer Sciences and Convergence Information Technology (ICCIT), 2010, pp. 836-839.

[49] IEEE std 802.15.4-2009, Part 15.4: Wireless Medium Access (mac) and Physical Layer (phy) Specifications for Low-Rate Wireless Personal Area Networks (wpans), 2009.

[50] C. Hsiang, A.W. Chen, C.J. Chang, B.Y. Shih, C.Y. Chen, Development of mechanisms for MAC channel selection to improve the performance of ieee 802.15.4 beacon-enabled network, in: 2010 3rd IEEE International Conference on Broadband Network and Multimedia Technology (IC-BNMT), 2010, pp. 598-602.

[51] Texas Instruments, CC2530 User Guide and Datasheet (accessed July 2013b).

[52] E. Casilari, J.M. Cano-Garca, G. Campos-Garrido, Modeling of current consumption in 802.15.4/zigbee sensor motes, MDPI Sensors 10 (6) (2010) 5443-5468.

[53] A. Koubaa, M. Alves, E. Tovar, A comprehensive simulation study of slotted CSMA/CA for IEEE802.15.4 wireless sensor networks, in: 2006 IEEE International Workshop on Factory Communication Systems, 2006, pp. 183-192.

[54] Linear Technology, LTC3108 Datasheet (accessed July 2014).

[55] Linear Technology, Ltspice iv - spice simulator <http:// www.linear.com/designtools/software/LTspice> (accessed July 2013).

[56] J.P. Amaro, J. Ferreira, R. Cortesão, J. Landek, A study on energy harvesting for wireless sensor networks using a split-core current transformer, in: Proceedings of Conference on Electronics Telecommunications and Computers, 2011b.

[57] J.P. Amaro, F.J.T.E. Ferreira, R. Corteso, J. Landeck, Energy harvesting for zigbee compliant wireless sensor network nodes, in: IECON 2012 - 38th Annual Conference on IEEE Industrial Electronics Society, 2012a.

[58] V. Sedlakova, J. Sikula, J. Majzner, H. Navarova, M. Chvatal, T. Zednicek, Tantalum and Niobium oxide capacitors: field crystallization, leakage current kinetics and reliability, in: 2010 27th International Conference on Microelectronics Proceedings (MIEL), 2010, pp. 439-442.

[59] BCcomponents, V. Aluminium Capacitors 150 CRZ Series, 2013

[60] Cellergy, Cellergy Capacitors, 2013.

[61] Texas Instruments, Z-Stack Sample Applications, Texas Instruments, San Diego, California, 2011a

[62] Texas Instruments, Z-Stack OS Abstraction Layer Application Programming Interface, Texas Instruments, 2011b.

[63] J.P. Amaro, J. Ferreira, R. Cortesão, J. Landek, Powering 802.15.4/ zigbee nodes on harvested energy, in: INForum 2012 Atas do 40 Simpósio de Informática, 2012b.

[64] J.P. Amaro, J. Ferreira, R. Cortesão, J. Landek, Powering wireless sensor networks nodes for complex protocols on harvested energy, in: Procedia Technology, vol. 5, 2012, 2012c.

[65] Z. Alliance, Zigbee Cluster Library Specificaton, 075123r02ZB, 2008 <http://www.zigbee.org/Specifications/ZigBee/download.aspx>

[66] I. Dietrich, F. Dressler, On the lifetime of wireless sensor networks, ACM Trans. Sen. Netw. 5 (1) (2009) 5:1-5:39, http://dx.doi.org/ 10.1145/1464420.1464425. <http://doi.acm.org/10.1145/1464420. 1464425>.

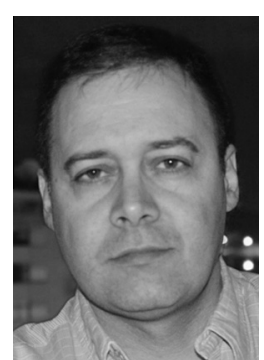

J. Pedro Amaro received the Licentiate degree in Electrical Engineering from the University of Coimbra in 1992 and the M.Sc. in Electronic System by Instituto Superior Técnico (IST), Technical University of Lisbon, Portugal in 2004. He is currently an Assistant Professor with the Department of Electrical Engineering, Engineering Institute of Coimbra (ISEC) of the Polytechnic Institute of Coimbra, Portugal. $\mathrm{He}$ is also a Research Fellow at the Institute of Systems and Robotics, University of Coimbra, where he is currently working in support of Ph.D. studies. Prior to this, he has worked at the Nuclear Fusion Center at the IST in Lisbon and the Lisbon Cadence Center where he worked in his M.Sc. thesis. His current research interests include wireless sensor networks and energy harvesting solutions. Also embedded systems development and FPGA based Digital Electronics are important topics that strongly relate to his academic and professional expertise.

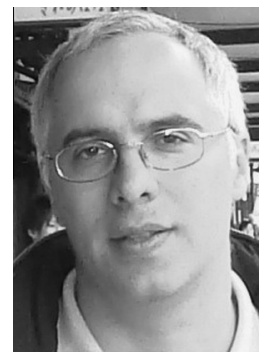

Rui Cortesão (M'05) received the B.Sc. ("Licenciatura") degree in Electrical Engineering, the M.Sc. degree in Systems and Automation, and the Ph.D. degree in Control and Instrumentation, all from the University of Coimbra, Coimbra, Portugal, in 1994, 1997, and 2003, respectively. He has been a Visiting Researcher with the German Aerospace Center - DLR (1998-2003), Stanford University (2002), and LIRMM-CNRS (2004-2006), where he was involved with compliant motion control, data fusion, steer-by-wire, haptic manipulation, and surgical robotics. He has been a Researcher with the Institute of Systems and Robotics (ISR-Coimbra) since 1994, and an Assistant Professor with the Electrical and Computer Engineering Department, University of Coimbra, where he teaches medical robotics and control. 


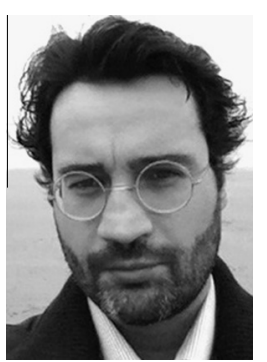

Fernando J.T.E. Ferreira (SM'09) received the Ph.D. degree in Electrical Engineering from the University of Coimbra, Coimbra, Portugal. He is currently a Professor in the Department of Electrical Engineering, Polytechnic Institute of Coimbra (IPC/ISEC), Coimbra, Portugal. Since 1998, he has been a Researcher in the Institute of Systems and Robotics, University of Coimbra (ISR-UC), working in the field of motors and drives. He has participated in several European projects dealing with energy efficient motor technologies. He is the coauthor of more than 50 papers published in international journals and conference records and presented at meetings. He was a recipient of the Best Paper Award at the 2001 IEEE/IAS Industrial and Commercial Power Systems Technical Conference, and of the Best Poster Presentation Award (for the technical competence displayed in the poster presentation) at the 2010 Inter. Conference on Electrical Machines.

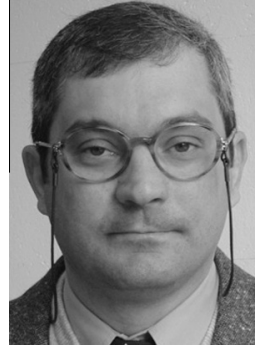

Jorge Landeck, b. 1967, Professor at the Science and Technology Faculty of the University of Coimbra and CTO of ISA - Intelligent Sensing. He owns a Ph.D. in Technological Physics and has more than 20 years experience in the areas of software, telemetry and distributed systems and instrumentation. He is author or co-author of several scientific publications in international refereed journals, managed several European projects and developed several innovative remote management systems. 